BNL-76997-2006-IR

\title{
Simulation of a Wide-Band Low-Energy Neutrino Beam for Very Long Baseline Neutrino Oscillation Experiments
}

Mary Bishai, et al.

August, 2006

\author{
Physics Department \\ Electronic Detector Group \\ Brookhaven National Laboratory \\ P.O. Box 5000 \\ Upton, NY 11973-5000 \\ www.bnl.gov
}

Notice: This manuscript has been authored by employees of Brookhaven Science Associates, LLC under Contract: No. DE-AC02-98CH10886 with the U.S. Department of Energy. The publisher by accepting the manuscript for publication acknowledges that the United States Government retains a non-exclusive, paid-up, irrevocable, world-wide license to publish or reproduce the published form of this manuscript, or allow others to do so, for United States Government purposes. 


\section{DISCLAIMER}

This report was prepared as an account of work sponsored by an agency of the United States Government. Neither the United States Government nor any agency thereof, nor any of their employees, nor any of their contractors, subcontractors, or their employees, makes any warranty, express or implied, or assumes any legal liability or responsibility for the accuracy, completeness, or any third party's use or the results of such use of any information, apparatus, product, or process disclosed, or represents that its use would not infringe privately owned rights. Reference herein to any specific commercial product, process, or service by trade name, trademark, manufacturer, or otherwise, does not necessarily constitute or imply its endorsement, recommendation, or favoring by the United States Government or any agency thereof or its contractors or subcontractors. The views and opinions of authors expressed herein do not necessarily state or reflect those of the United States Government or any agency thereof. 


\title{
Simulation of a Wide-Band Low-Energy Neutrino Beam for Very Long Baseline Neutrino Oscillation Experiments
}

\author{
Mary Bishai ${ }^{1}$, Jordan Heim ${ }^{2}$, Christine Lewis ${ }^{3}$, \\ Alysia D. Marino ${ }^{4}$, Brett Viren ${ }^{2}$, Francisco Yumiceva ${ }^{5}$ \\ ${ }^{1}$ Brookhaven National Labonatory, P.O. Box 5000, Upton, NY 11950, USA \\ 2 Purdue University, Department of Physics, 535 Northwestem Avenue, West Lafayette, IN 47907 , USA \\ ${ }^{3}$ Columbia University, Department of Physics, 538 West 120th. Street, New York, NY 10027, USA \\ 4 Fermi National Accelerator Laboratory, P.O. Box 500, Batavia, IL 60510, USA \\ 5 The College of William and Mary, P.O. Box 8795, Williamsburg, VA 23187, USA
}

(Dated: July 28, 2006)

\begin{abstract}
We present simulations of a wide-band low-energy neutrino beam for a future very long baseline neutrino oscillation (VLBNO) program using the proton beam from the Main Injector (MI) proton accelerator at Fermi National Accelerator Laboratory (Fermilab). The target and horn designs previously developed for Brookhaven Laboratory's Alternating Gradient Synchrotron (AGS) VLBNO program are used without modifications. The neutrino flux distributions for various MI proton beam energies and new high-intensity neutrino beam-line designs possible at Fermilab are presented. The beam-line siting and design parameters are chosen to match the requirements of an on-axis beam from Fermilab to one of the two possible sites for the future Deep Underground Science and Engineering Laboratory (DUSEL). A preliminary estimate of the observable event rates and spectra at a detector located in DUSEL for different beam configurations has been performed. Our preliminary conclusions are that a $40-60 \mathrm{GeV} 0.5$ to $1 \mathrm{MW}$ beam from the Fermilab Main Injector to a DUSEL site has the potential to reach the desired intensity for the next generation of neutrino oscillation experiments. Recent studies indicate that the Fermilab MI can reach a beam power of $0.5 \mathrm{MW}$ at $60 \mathrm{GeV}$ with incremental upgrades to the existing accelerator complex.
\end{abstract}




\section{Contents}

I. Introduction

3

II. Prospects for siting a new high-intensity neutrino beam-line at Fermi National Lab

III. Simulation of a Wide-Band Low-Energy Beam

A. Target simulation

B. Horn simulation

C. Target region layout and geometry

D. Comparative studies with NuMI and AGS simulations

IV. Optimization of the Fermilab WBLE beam spectrum

V. Event Rates at the Far Detector

VI. Summary

Acknowledgments

A. Horn current optimization studies

References 


\section{INTRODUCTION}

The physics case for very long baseline neutrino oscillation (VLBNO) experiments using an on-axis wide-band neutrino beam has been presented in [1], [2]. It has previously been demonstrated that an on-axis wide-band neutrino beam directed at a few 100 kilo ton scale Water Cerenkov neutrino detector located at a baseline greater than $1000 \mathrm{~km}$ can provide a powerful probe of the parameters of the neutrino mixing matrix, the mass hierarchy, and CP-violation.

The Main Injector (MI) $120 \mathrm{GeV}$ proton accelerator at Fermi National Accelerator Laboratory (Fermilab) is an attractive candidate as the source of a neutrino beam for VLBNO experiments. The longest baseline neutrino oscillation experiment currently in operation is the Main Injector Neutrino Oscillation Search (MINOS) experiment based at Fermilab which utilizes the NuMI (Neutrinos at the Main Injector) [3] beam-line from the MI. The NuMI beam-line has been operational since Jan 21,2005 and has delivered $1.4 \times 10^{20}$ protons-ontarget (POT) to the MINOS experiment in its first year of operation [4]. The GEANT [5] based simulation of the NuMI beam-line has now been validated using real data from the MINOS experiment. The NuMI simulation software has proven to be a remarkable success at predicting the measured neutrino charged-current (CC) interaction rates observed in the MINOS near detector. The level of agreement between the data and simulation CC interaction rates is within $10 \%$ in the region of interest for the MINOS experiment.

In this study we will focus on simulating the performance of a proposed new neutrino beam-line for a VLBNO program based at Fermilab using the $120 \mathrm{GeV}$ Main Injector (MI) proton accelerator. We will use the NuMI simulation framework and incorporate the target and horn simulations that had been previously developed for the VLBNO program at

Brookhaven National Lab's (BNL) Alternating Gradient Synchrotron (AGS) [6] to predict the neutrino fluxes produced from a wide-band low-energy (WBLE) beam using the Fermilab MI.

The design specifications of a new WBLE beam based at the Fermilab MI are driven by 


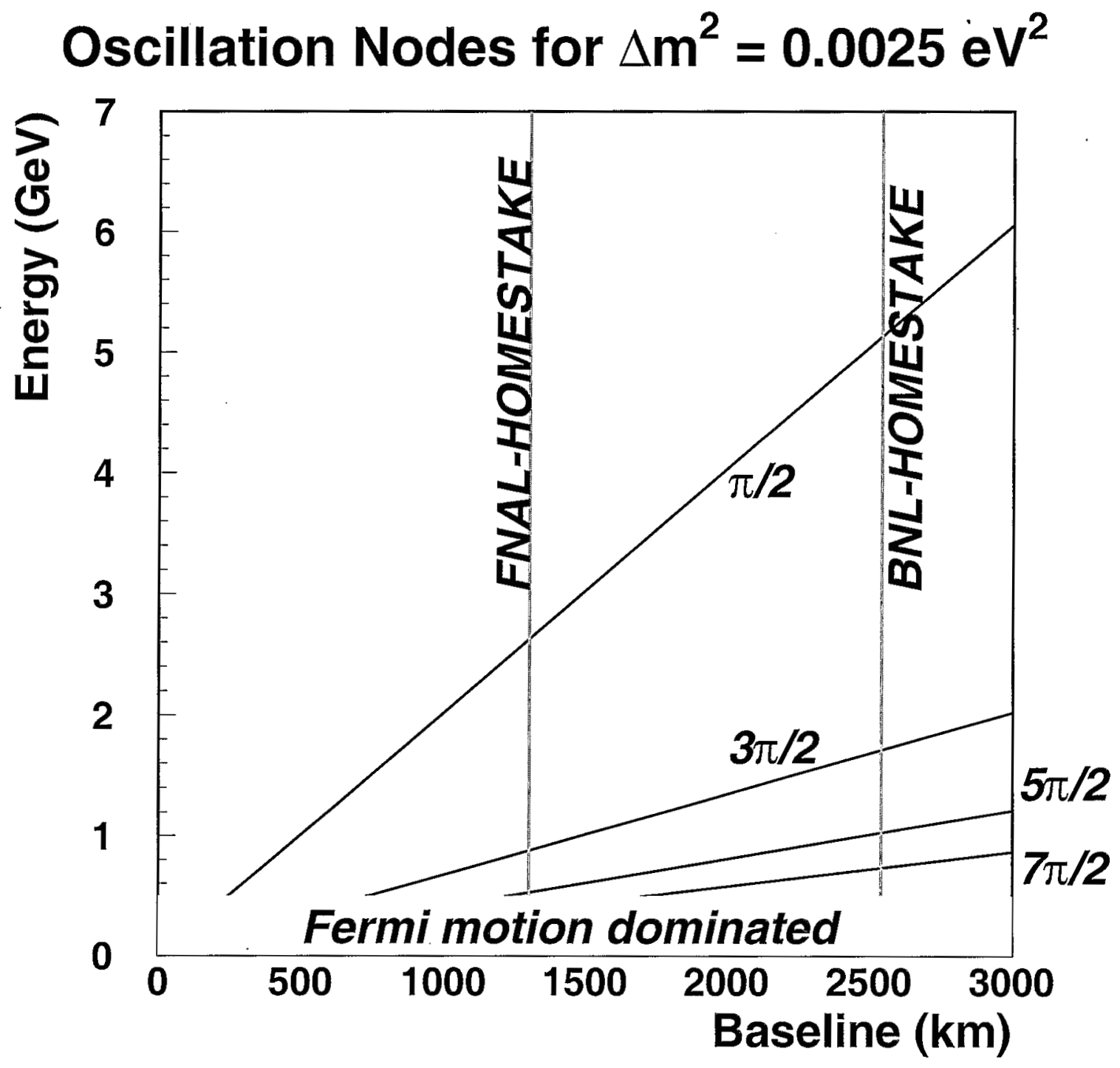

FIG. 1: Location of oscillation nodes in the neutrino energy spectrum as a function of distance from the beam source.

the physics of $\nu_{\mu} \rightarrow \nu_{\mu}$, and $\nu_{\mu} \rightarrow \nu_{e}$ oscillations. In Figure 1, the locations of the oscillation maxima as a function of distance from the neutrino source - the baseline - and the neutrino energy are displayed. In principle, the ideal neutrino beam for the next generation neutrino program envisioned in [1] and [2] would be one that has a wide energy band that covers the energy region from low energies to the energy of the first $(\pi / 2)$ oscillation maximum 
and minimal flux beyond the region of interest. Low flux at high neutrino energies is desired to eliminate neutral-current backgrounds from high energy neutrinos that are not sensitive to oscillations but still produce significant background at low observed energies in the neutrino detectors. The oscillation spectrum looks different at different baselines, therefore, for different baselines, the ideal beam energy spectrum which maximizes the sensitivity to oscillations will have different properties. For the purpose of this study, we will consider the two proposed sites for the Deep Underground Science and Engineering Laboratory (DUSEL) [7] as possible locations for the far neutrino detector using Fermilab as the neutrino beam source. The two proposed DUSEL sites are The Homestake Mine, SD and The Henderson Mine, CO. These two sites are located at a baseline of $1297 \mathrm{Km}$ and $1487 \mathrm{Km}$ from Fermilab respectively.

In this study we will concentrate on optimizing the neutrino beam for the VLBNO program from Fermilab to The Homestake Mine (FNAL-HS). Since the distances from Fermilab to Homestake and Henderson Mines are not significantly different, the same beam design will be applicable for both baselines. We specify the following broad requirements for a FNAL-HS beam based on examination of the oscillation nodes in Figure 1:

1. We require the maximal possible neutrino fluxes to encompass at least the 1st and 2 nd oscillation nodes, the maxima of which occur at 2.4 and $0.8 \mathrm{GeV}$ respectively.

2. Since neutrino cross-sections scale with energy, larger fluxes at lower energies are desirable to achieve the physics sensitivities using effects at the 2nd oscillation node and beyond.

3. To detect $\nu_{\mu} \rightarrow \nu_{e}$ events at the far detector, it is critical to minimize the neutralcurrent contamination at lower energy, therefore minimizing the flux of neutrinos with energies greater than $5 \mathrm{GeV}$ where there is little sensitivity to the oscillation parameters (including the CP phase and the mass hierarchy) is highly desirable.

4. The irreducible background to $\nu_{\mu} \rightarrow \nu_{e}$ appearance signal comes from beam generated 
$\nu_{e}$ events, therefore, a high purity $\nu_{\mu}$ beam with as low as possible $\nu_{e}$ contamination is required. 


\section{PROSPECTS FOR SITING A NEW HIGH-INTENSITY NEUTRINO BEAM-}

\section{LINE AT FERMI NATIONAL LAB}

The current conceptual layout for siting a new neutrino beam-line from Fermi National Lab to the two proposed DUSEL sites. [8] [9] is shown in Figure 2. The siting layout

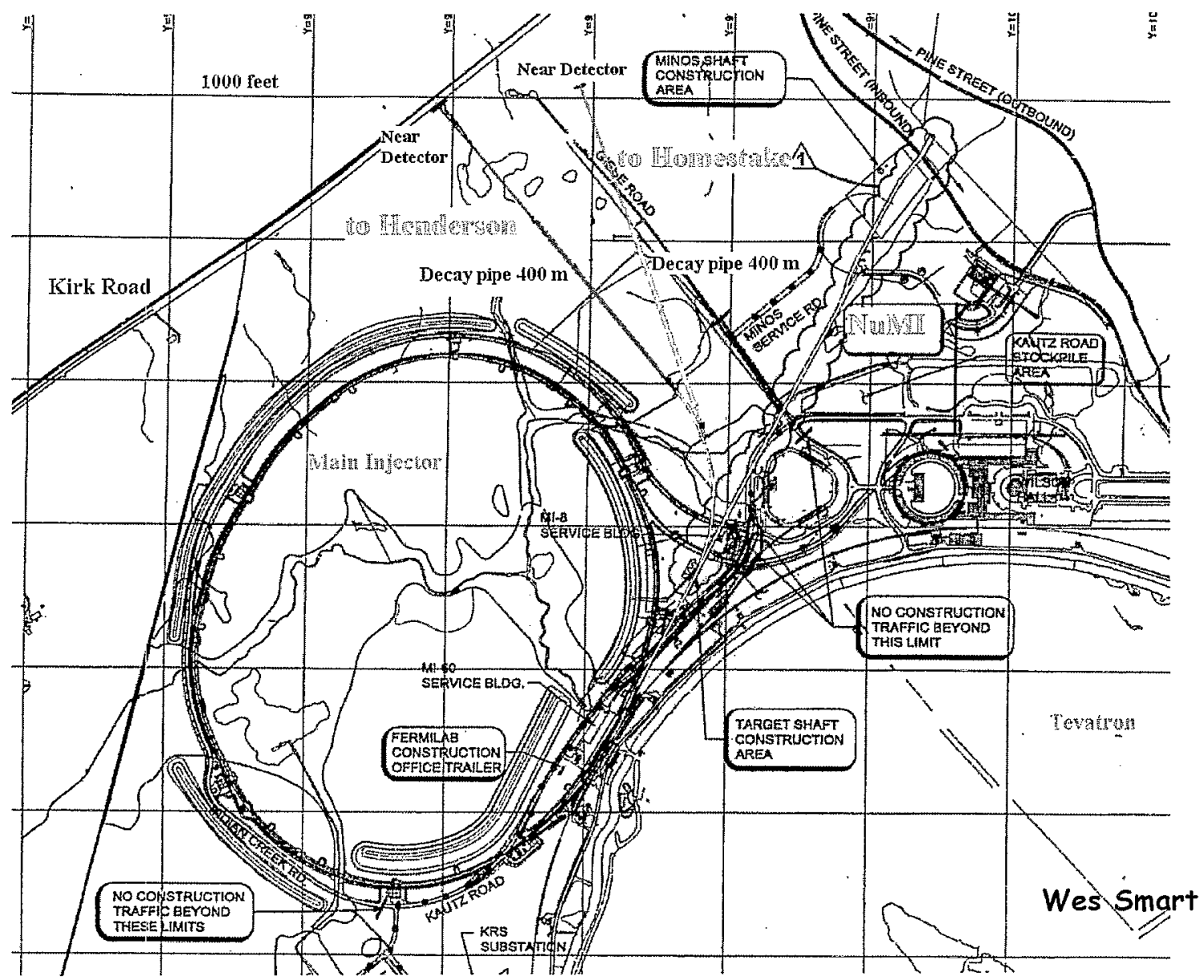

FIG. 2: Possible siting of a long baseline neutrino beam-line from the Fermilab site to the two current proposed sites for DUSEL [8].

shown in Figure 2 [10] has the following conceptual design properties:

- The present extraction of the Main Injector into the NuMI beam-line will be used.

- An additional tunnel will be constructed starting from the approximate location of the NuMI lower Hobbit door, at the end of the carrier tunnel (see Figure 3), in order 


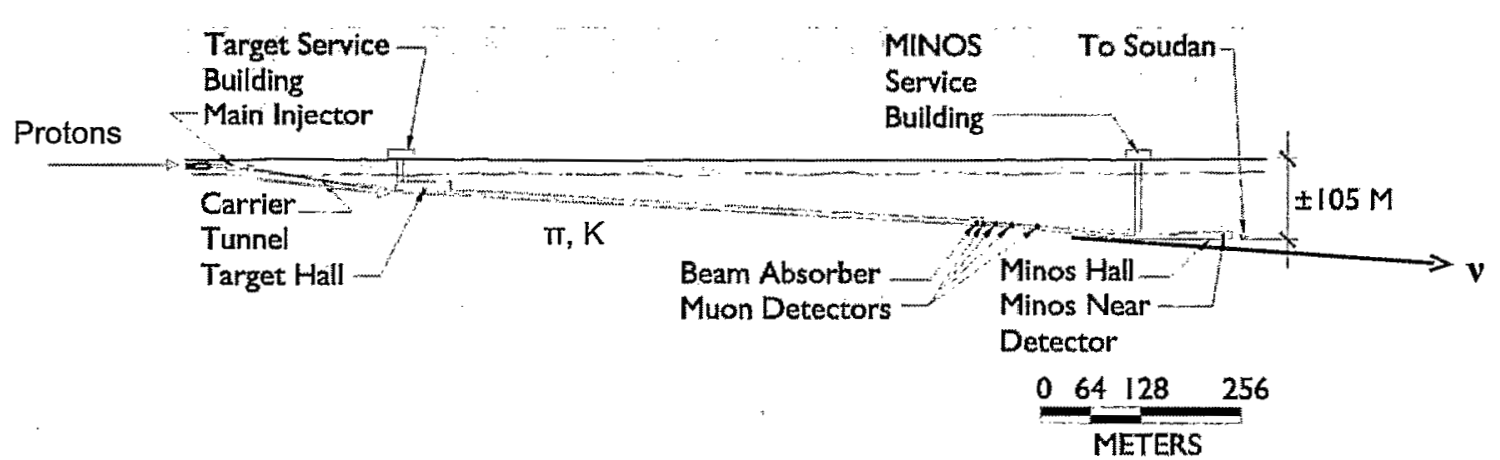

FIG. 3: Elevation view of the NuMI beamline.

to transport the proton beam to the west.

- The radius of curvature of the tunnel bending to the west will be similar to the Main Injector curvature which will enable protons with energies up to $120 \mathrm{GeV}$ to be steered along the bend using conventional magnets.

- The target hall length is $\leq 45 \mathrm{~m}$.

- A decay tunnel length of up to $400 \mathrm{~m}$ can be accommodated on the site assuming the near detector is $300 \mathrm{~m}$ from the end of the decay pipe as in NuMI. A shorter decay pipe than the NuMI decay pipe $(677 \mathrm{~m})$ may be desirable to limit the production of higher energy neutrinos.

- The low energy neutrino flux can be enhanced by increasing the decay pipe radius. The widest span in the NuMI beam-line is the MINOS detector hall width at $37 \mathrm{ft}$ wide, which is at the limit possible in the Fermilab rock. For a $\leq 2 \mathrm{MW}$ beam the concrete shielding needed around the decay pipe increases from the 4 feet used in the $400 \mathrm{~kW}$ NuMI beam-line to about 8 feet [10]. If we assume an extra 1.m of clearance left between the decay pipe and tunnel, we find that decay pipes of up to $2.3 \mathrm{~m}$ in radius may be accommodated. Larger decay pipes may be possible if more expensive thinner steel shielding is used instead of concrete. 
Given the conceptual design parameters of the FNAL-DUSEL neutrino beam-line outlined above, we will optimize the neutrino energy spectrum by varying the decay pipe widths up to $2 \mathrm{~m}$ in radius and $400 \mathrm{~m}$ in length. The BNL AGS neutrino beam used for the VLBNO program proposed at BNL [6] utilized a decay pipe of $2 \mathrm{~m}$ radius and $180 \mathrm{~m}$ in length.

The time needed for a VLBNO experiment from Fermilab to a DUSEL site to reach the design sensitivity will depend on the neutrino flux in the oscillation region, the neutrino beam power, and the size of the far detector. The conceptual design of a 2 MW High Energy Neutrino Source (HINS) at FNAL using the Proton Driver concept is explored in [11]. Recently, several studies at Fermilab [12] have indicated that the accelerator complex has the ability to increase the beam power up to $1 \mathrm{MW}$ at $120 \mathrm{GeV}$ without the need for a Proton Driver. The variation in beam power that could be delivered by the existing complex as a function of beam energy is driven by: 1) the proton beam intensity that can be injected into the Main Injector each cycle, and 2) the Main Injector cycle time. In Figure 4, the MI ramp times at different proton energies are shown.

In addition to the decrease in MI cycle time at lower beam energies, the possibility of using both the existing recycler and anti-proton accumulator storage rings to accumulate protons from the $8 \mathrm{GeV}$ booster during the MI cycle is being explored. This scenario has the potential of increasing the proton beam intensity injected into the MI each cycle to the maximum the current machine can handle $\left(6 \times 10^{13}\right.$ protons $)$ with incremental upgrades to the existing complex. The beam power as a function of beam energy predicted for different upgrade possibilities to the accelerator complex is shown in Figure 5.

We find that in the $40-60 \mathrm{GeV}$ range, a beam power of approximately $0.5 \mathrm{MW}$ is possible with incremental changes to the current accelerator complex. Since the neutrino flux will increase with beam energy, it is one of the goal of these studies to determine whether the same physics sensitivities previously obtained with the $28 \mathrm{GeV}$ 1MW AGS beam using a $180 \mathrm{~m}$ long decay pipe with a $2 \mathrm{~m}$ radius [1] [2] may be achievable with a Fermilab $0.5 \mathrm{MW}$ $60 \mathrm{GeV}$ beam, and a wider, longer decay pipe. 


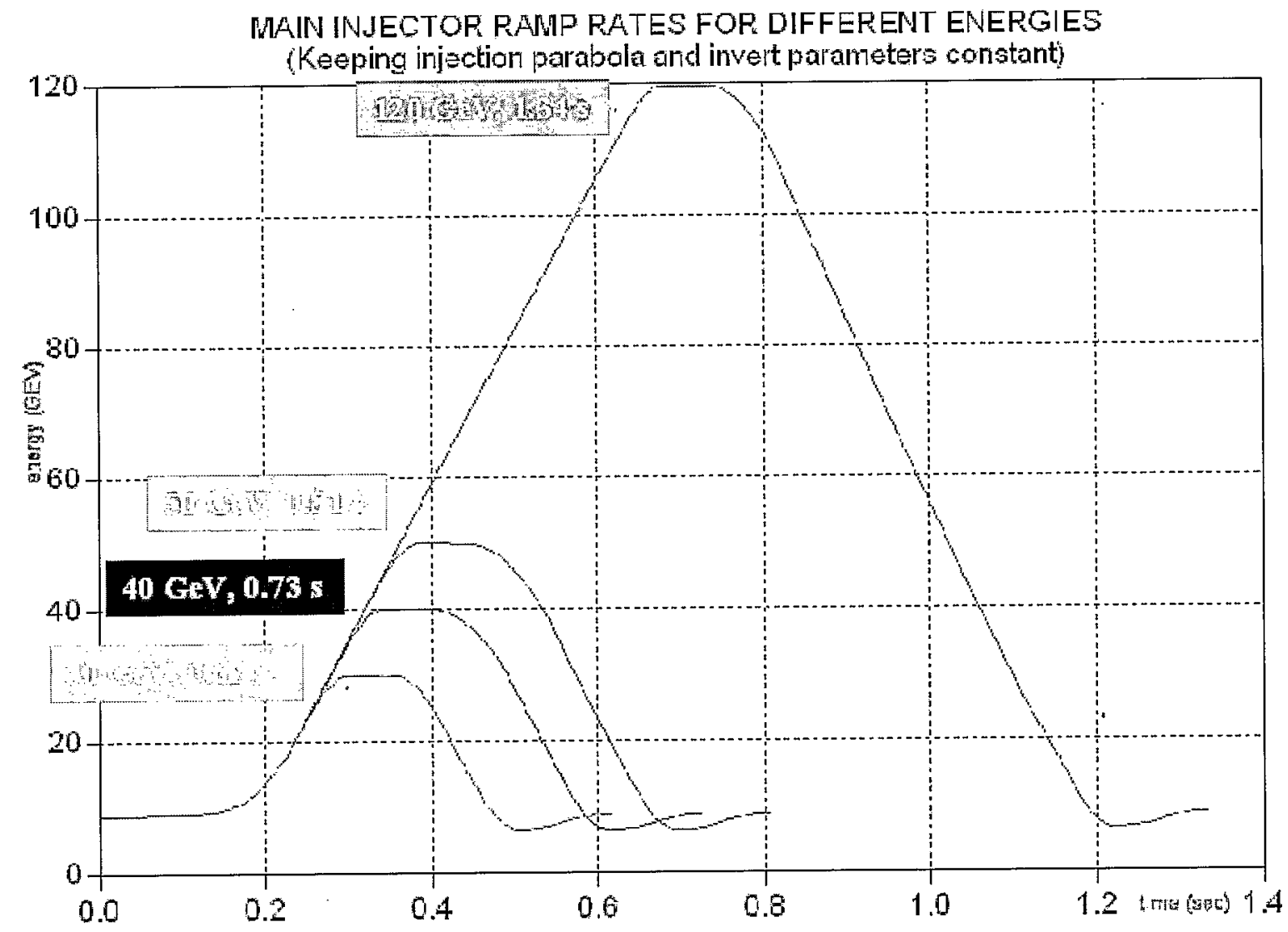

FIG. 4: Main injector ramp times at different proton beam energies [8]. 


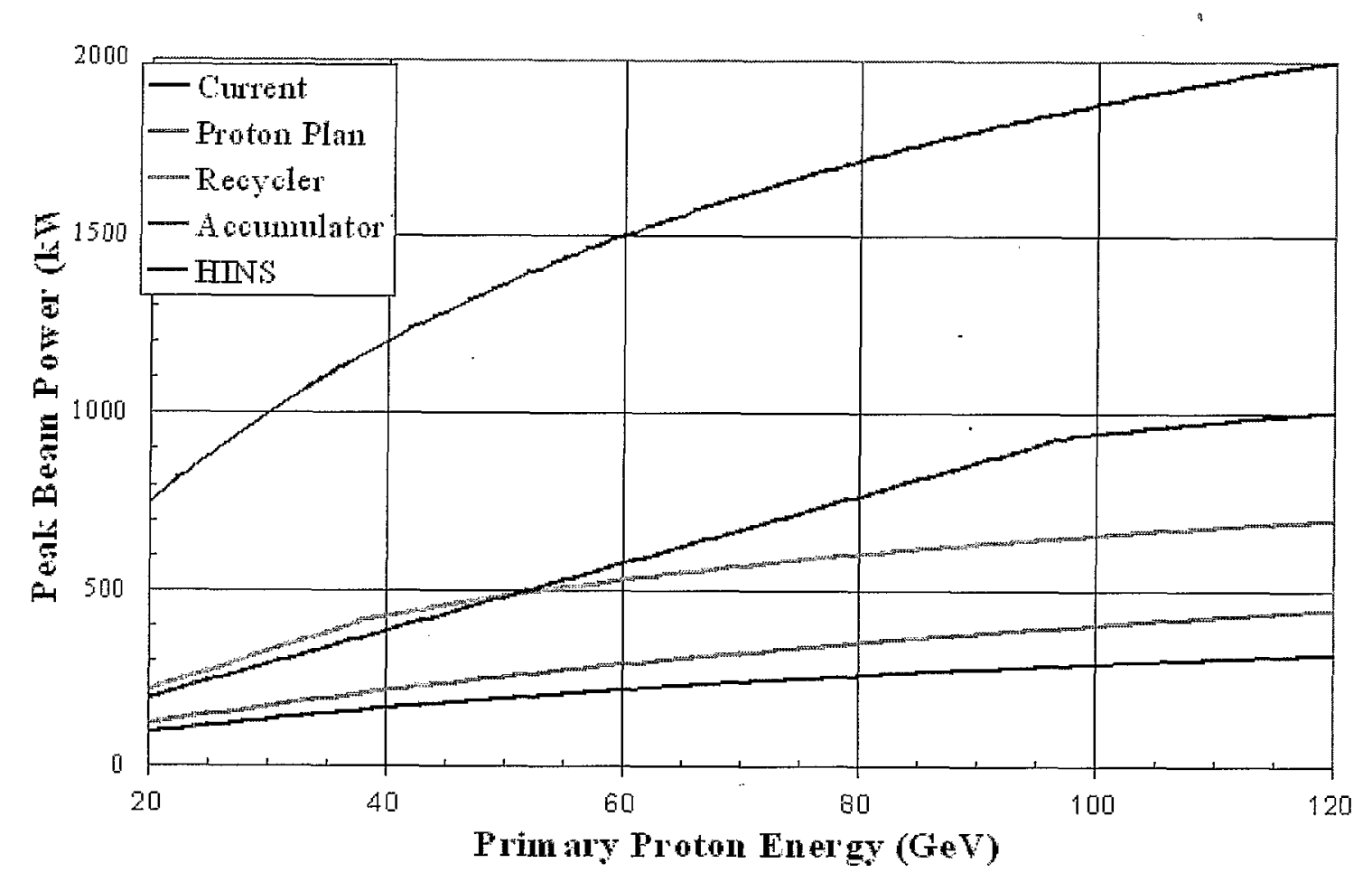

FIG. 5: Projections of main injector proton beam power as a function of beam energy for different upgrade scenarios under consideration at Fermi National Lab [12]. The proton-plan refers to the current studies underway to slip-stack proton batches from the booster into the MI, thus increasing the number of protons per MI batch. The recycler and accumulator plans involve the use of the recycler and anti-proton accumulator to accumulate booster protons during the MI cycle and then inject from recycler/accumulator into the MI. This scenario becomes possible after the Tevatron shutdown. The HINS is the upgrade to 2 MW using a Super Conducting Linac (SCL) to replace the booster and significant upgrades to the MI. 


\section{SIMULATION OF A. WIDE-BAND LOW-ENERGY BEAM}

In Figure 6, the spectrum of reconstructed charged-current (CC) neutrino interactions measured in the MINOS near detector is compared to the Monte Carlo simulation (MC) results. Both data and $\mathrm{MC}$ are absolutely normalized to the number of protons delivered to the NuMI beam-line. We find that the NuMI/MINOS simulation predicts the absolute neutrino rate observed in the MINOS detector in the 0-7 GeV region to better than $10 \%$. In the high energy region of $>10 \mathrm{GeV}$, the disagreement between simulation and data is around 30\%. The preliminary studies from MINOS [4] indicate that a large fraction of the discrepancy between data and MC at large neutrino energies can be attributed to the uncertainty on the hadro-production models that predict the hadron spectrum produced when the proton beam interacts with the NuMI graphite target. These results from the MINOS experiment provide us with invaluable quantitative information on how well the NuMI beam simulation predicts absolite neutrino rates in a working experiment.

Using the NuMI simulation framework, we have produced a preliminary simulation of a wide-band low-energy (WBLE) beam. In this section we discuss the implementation of the target and horn design for a WBLE neutrino beam in the NuMI simulation framework.

\section{A. Target simulation}

The NuMI beam simulation uses FLUKA '05 [13] to generate the hadron spectrum from the interaction of the NuMI proton beam with a graphite target. Comparisons of data on inclusive particle production at the AGS in $14 \mathrm{GeV}$ proton-nucleus collisions with several simulations [14] have concluded that FLUKA '05 produces the best overall agreement with the data. The NuMT target design is described in [3]. The NuMJ target is composed of $4720 \mathrm{~mm}$ long graphite segments with $0.3 \mathrm{~mm}$ spacing. The dimensions of each segment transverse to the proton beam are $6.4 \mathrm{~mm}$ in width and $18 \mathrm{~mm}$ in height. The target density used is $1.784 \mathrm{~g} / \mathrm{cm}^{3}$. The target is water cooled and is 1.9 proton interaction lengths long. The NuMI target geometry simulated in FLUKA 05 is shown in Figure 7. In Figure 8, the 

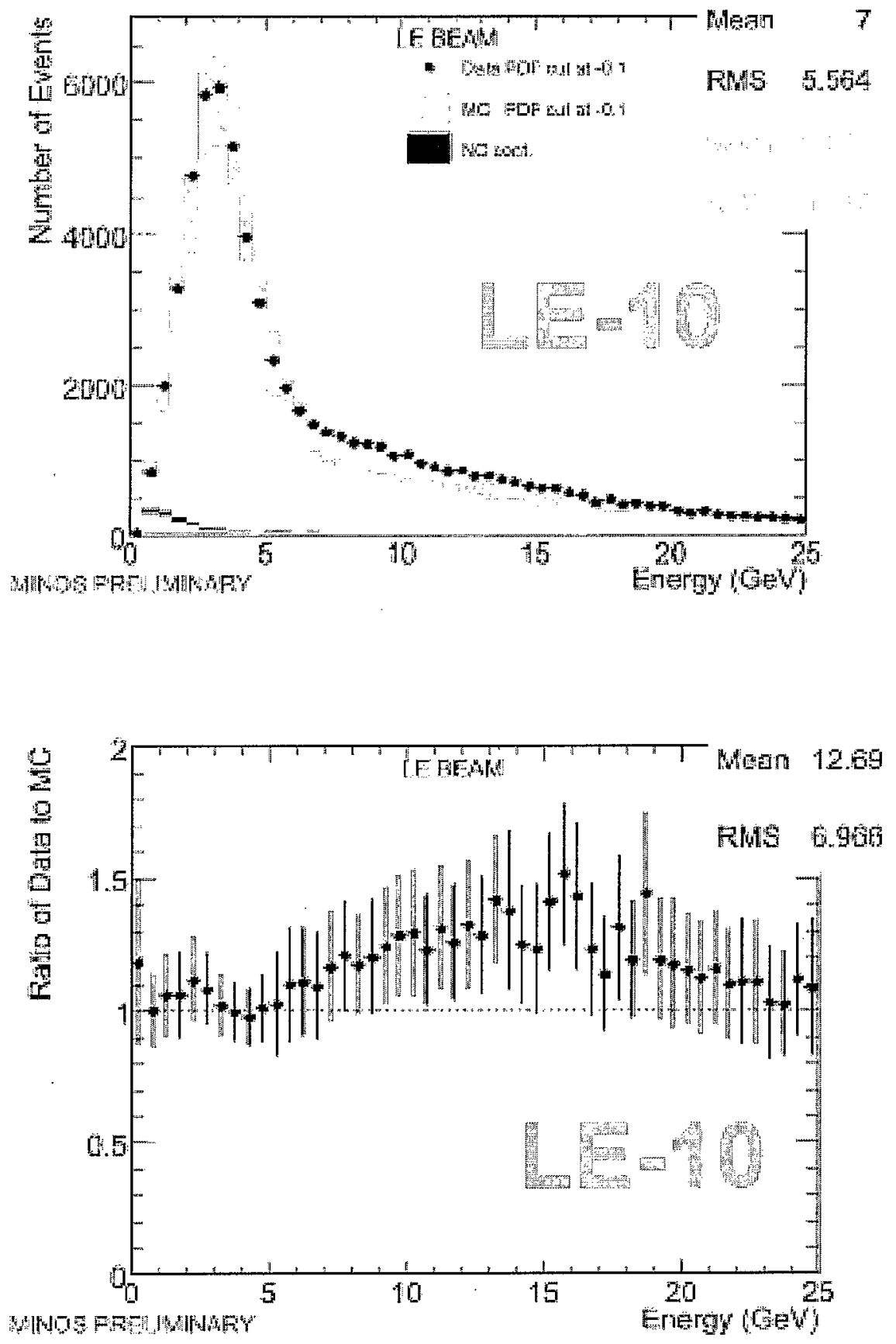

FIG. 6: Comparison between the MINOS near detector measured CC spectrum and the GEANT simulation of the NuMI beam using Fluka, 2005 for hadro-production on the target.

measurements of the proton beam RMS in the NuMI pre-target region as a function of NuMI beam intensity per batch [20] are plotted. For the data collected from May, 2005 through 


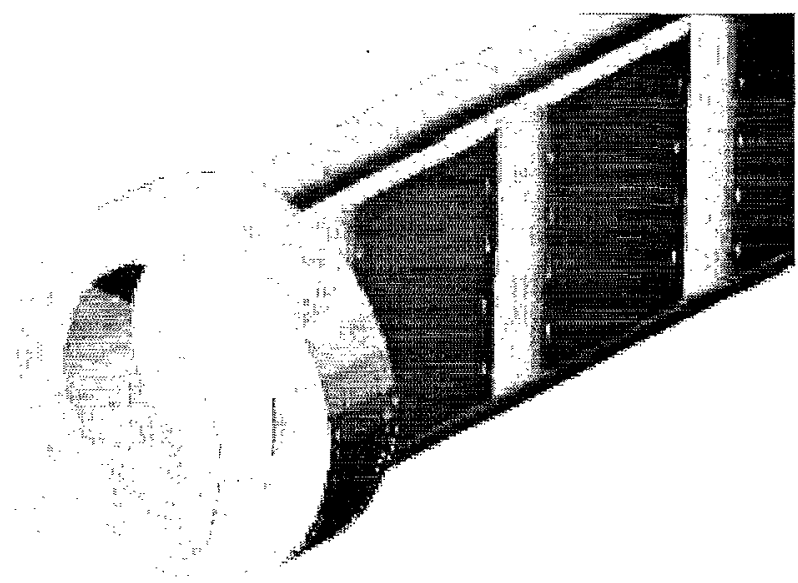

FIG. 7: NuMI target geometry as implemented in Fluka05. The target segments are green, the cooling tubes are shown in yellow and the Be window is shown in magenta.

\section{NuMI beam profiles versus average batch intensity}

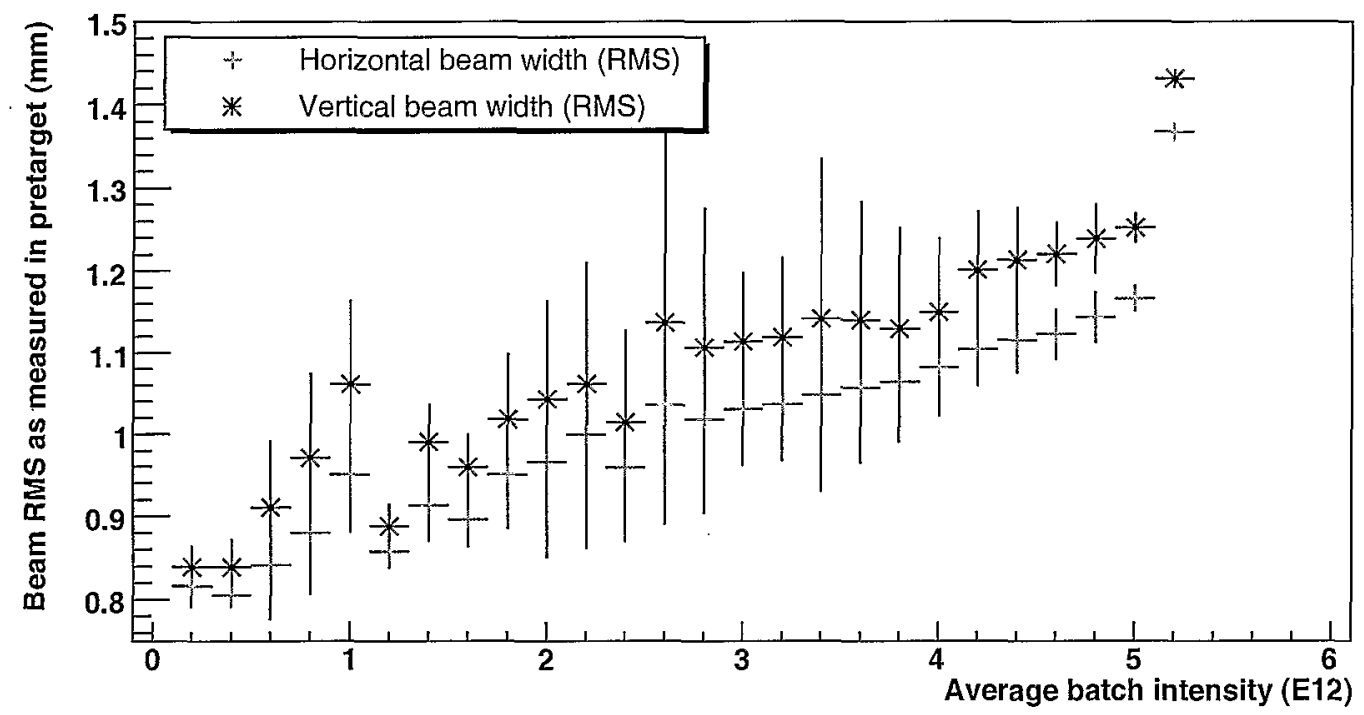

FIG. 8: NuMI beam profile as a function of batch intensity. The points are the mean beam RMS and the error bars represent the spread at each batch intensity. The last point is from the first high intensity proton pulse delivered to NuMI using slip-stacked bunches. At this time slip stacking is still in study at NuMI and the beam profile measured for the slip-stacked pulse may not be representative of the beam profiles from well controlled slip-stacked pulses.

Feb, 2006, the average NuMI beam intensity was 4-5 E12 protons/batch which corresponds 
to a proton mean beam RMS of $1.1 \mathrm{~mm}$ in the horizontal and $1.25 \mathrm{~mm}$ in the vertical. These measurements are used in the FLUKA '05 simulation of the NuMI proton beam. The proton beam energy used in the NuMI simulation is $120 \mathrm{GeV}$.

For the WBLE simulation we use a target geometry based on the BNL AGS study of a WBLE neutrino beam [6] which is described in detail in [15]. The WBLE target is a cylindrical rod of a carbon-carbon (CC) composite material, $80 \mathrm{~cm}$ in length with a $12 \mathrm{~mm}$ diameter. The density of the target is $2.1 \mathrm{~g} / \mathrm{cm}^{3}$. The current AGS target design is cooled by circulating forced Helium gas, although water cooling is also under consideration. The FLUKA '05 implementation of a simple graphite rod bathed in Helium is shown in Figure 9. The maximum proton intensity that the current Fermilab MI can handle is around 60 E12 protons (10 E12/batch) at $120 \mathrm{GeV}$. Using the NuMI beam as a guideline and extrapolating from Figure 8 , we use a proton beam RMS of $1.5 \mathrm{~mm}$ horizontally and vertically in the FLUKA '05 WBLE target simulation. We simulated hadro-production from the WBLE target using different proton beam energies varying from 28 to $120 \mathrm{GeV}$. For simplicity, the beam RMS used was the same for all proton energies simulated.

Table I summarizes the properties of the NuMI and WBLE target design and beam parameters used in the FLUKA '05 and GEANT simulation.

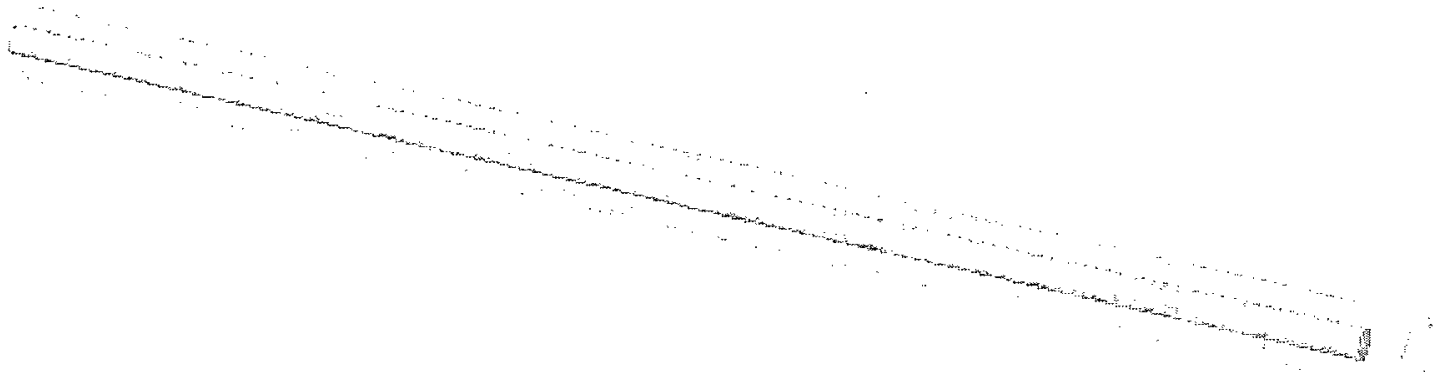

FIG. 9: WBLE target geometry as implemented in Fluka05 
TABLE I: Target and beam parameters: NuMI and WBLE

\begin{tabular}{lcc}
\hline Component & NuMI & WBLE \\
\hline Shape: & 47 rectangular segments & solid cylindrical rod \\
& each $6.4 \mathrm{~mm}$ wide $\times 18 \mathrm{~mm}$ high & 12mm diameter \\
& and $20 \mathrm{~mm}$ long & \\
& $=0.954 \mathrm{~m}$ total length & $0.8 \mathrm{~m}$ total length \\
Material: & graphite & carbon-carbon composite \\
Density: & $1.784 \mathrm{~g} / \mathrm{cm}^{3}$ & $2.1 \mathrm{~g} / \mathrm{cm}^{3}$ \\
Cooling: & water cooling tubes & Helium flow cooled \\
\hline & Proton beam parameters \\
Energy: & $120 \mathrm{GeV}$ & $28,40,60,120 \mathrm{GeV}$ \\
RMS width: & $\sigma_{x}=1.1 \mathrm{~mm}, \sigma_{y}=1.25 \mathrm{~mm}$ & $\sigma_{x}=1.5 \mathrm{~mm}, \sigma_{y}=1.5 \mathrm{~mm}$ \\
\hline
\end{tabular}




\section{B. Horn simulation}

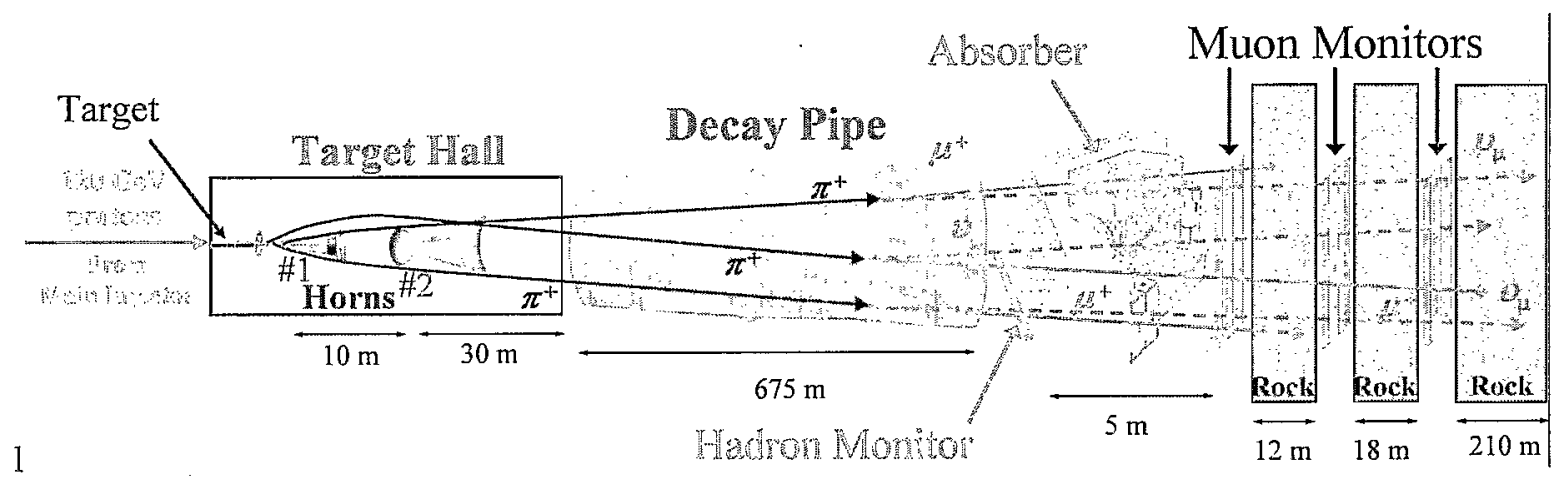

FIG. 10: Schematic of the NuMT beam-line

The NuMI beam uses two parabolic magnetic focusing horns to focus positively charged hadrons produced in the target in the momentum range of $1-10 \mathrm{GeV} / \mathrm{c}$ into a $677 \mathrm{~m}$ long $1 \mathrm{~m}$ radius decay pipe, as shown in Figure 10. In the NuMI simulation, GEANT version 3.21 $[5]$ is used to swim the hadrons, produced by a FLUKA '05 simulation of proton interactions in the target, through the focusing horns, the decay region, and the final hadron absorber. The NuMI horn shapes are double parabolic. The GEANT implementation of the NuMI horns is shown in Figure 11. The distance between the upstream end of Horn 1 and the upstream end of Horn 2 is $10 \mathrm{~m}$. The NuMI horn design parameters implemented in GEANT are summarized in Table II. Figure 11 shows the path of hadrons produced in the target by several $120 \mathrm{GeV}$ protons incident from the left as they propagate through the NuMI horns. In this simulation the horn current is set at $185 \mathrm{kA}$ [4].

In the current implementation of the GEANT simulation of the WBLE horns, we have used the horn geometry from the BNL AGS study [6] without further optimization. The WBLE horn parameters used in this study are summarized in Table II. This horn geometry was optimized for use with a $28 \mathrm{GeV}$ proton beam and may not be optimal for use with the higher beam energies available from the Fermilab MI. Studies are currently underway to further optimize the WBLE horn design to better match the MI beam energies. We have used the NuMI GEANT implementation and replaced the NuMI horns with the WBLE horns. 

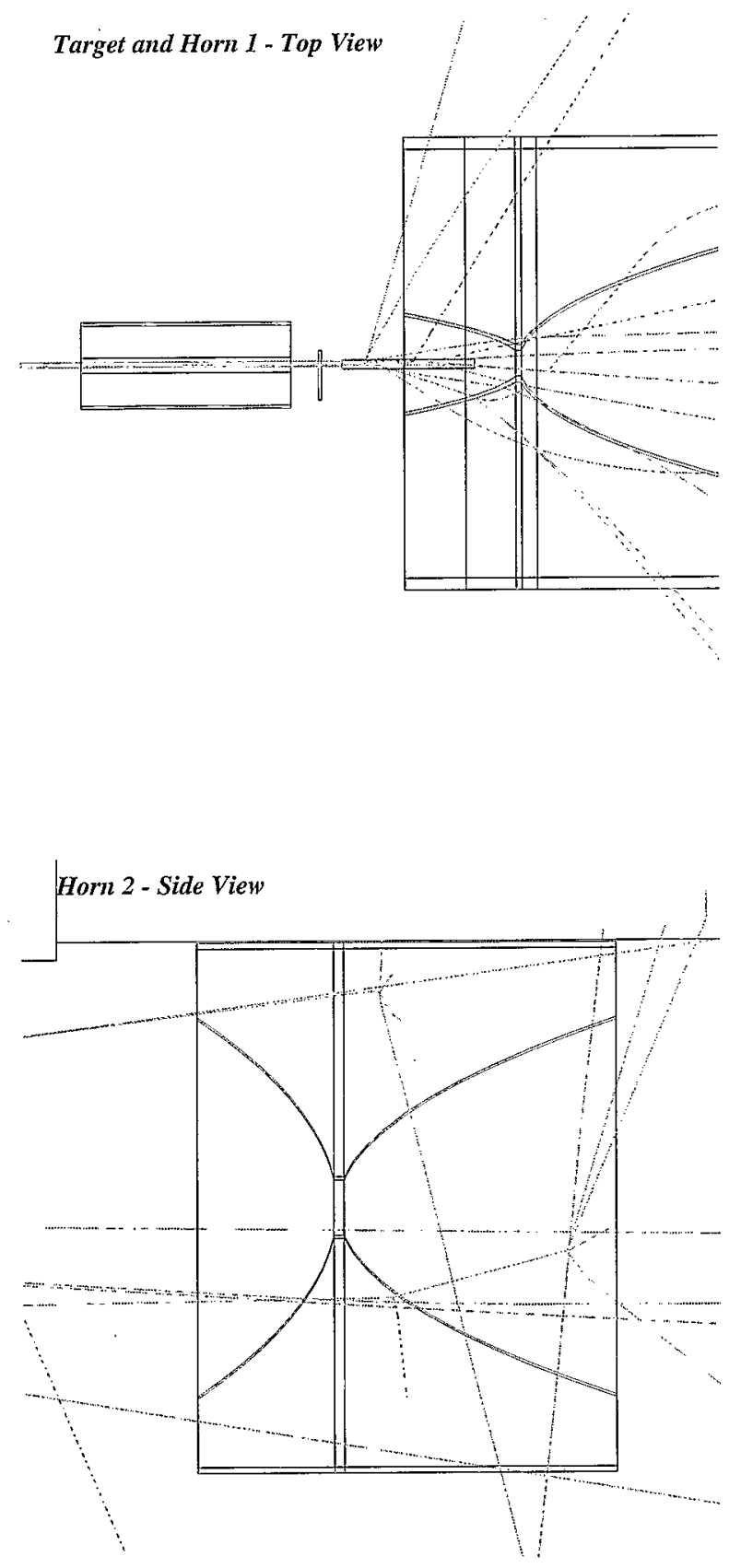

FIG. 11: GEANT simulation of the NuMI target and horns with $120 \mathrm{GeV}$ p+. The Horn 1 crosssection is shown on top and horn 2 is shown on the bottom. The Nu.MI target is $1 / 2$ way into the front of horn 1. The vertical and horizontal scales are in the ratio of 1 to 10 (top) and 1 to 5 (bottom). The beam is incident from the left. The horn current used in this simulation is $185 \mathrm{kA}$. 

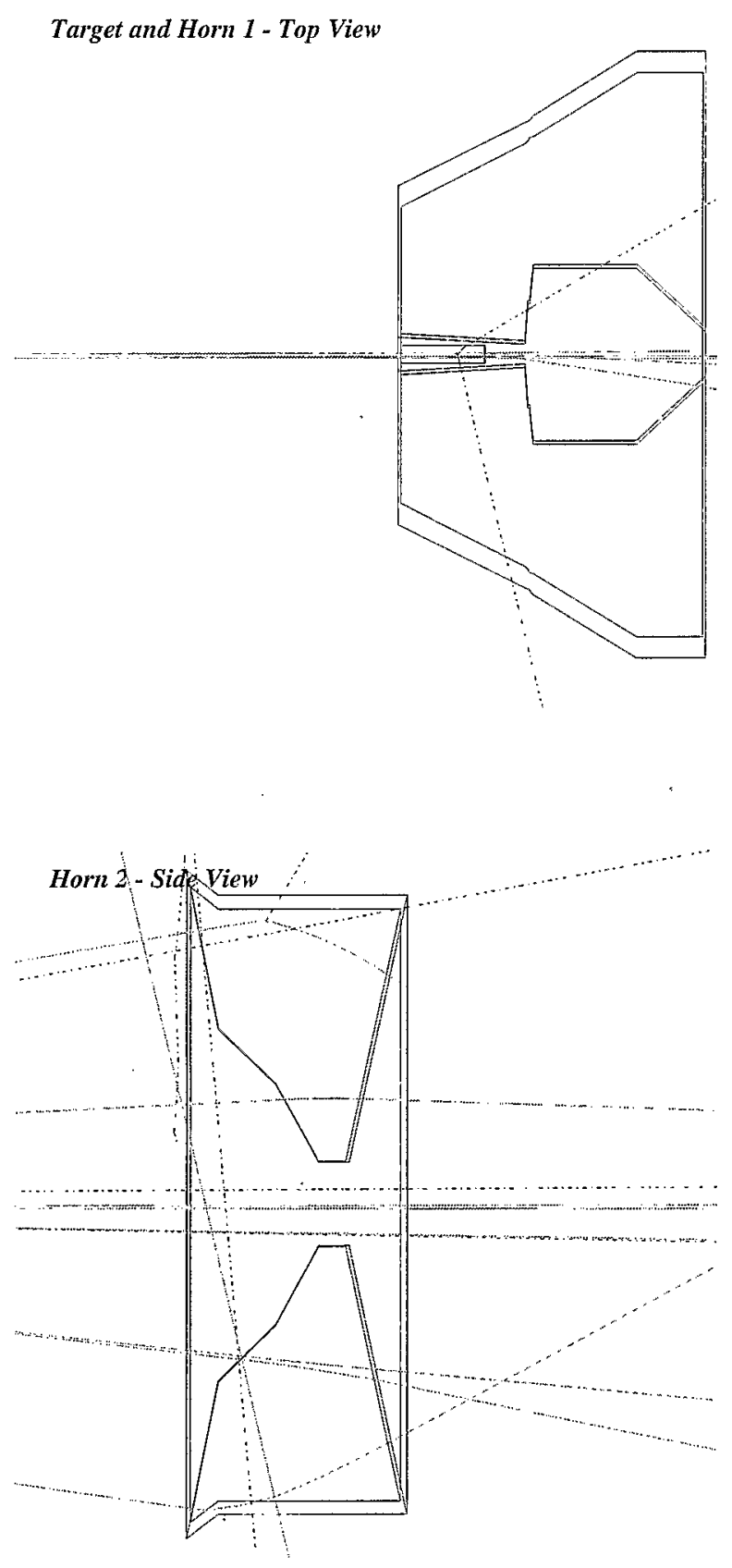

FIG. 12: GEANT simulation of the WBLE target and horns with $120 \mathrm{GeV}$ p + . The Horn 1 crosssection is shown on top and horn 2 is shown on the bottom. The WBLE target is fully contained within horn 1. The vertical and horizontal scales are in the ratio of 1 to 10 (top) and 1 to 5 (bottom). The beam is incident from the left. The horn current used in this simulation is $185 \mathrm{kA}$. 
The WBLE horn geometry as it has been implemented in the NuMI GEANT framework is shown in Figure 12. The distance between the upstream end of Horn 1 and the upstream end of Horn 2 is $10 \mathrm{~m}$. Figure 11 shows the path of hadrons produced in the target by several $120 \mathrm{GeV}$ protons incident from the left as they propagate through the WBLE horns. In this simulation the horn current is set at $185 \mathrm{kA}$ [4] for easy comparison to NuMI. We intend to optimize the WBLE horn current as part of this study. The baseline design current is set at a maximum of $250 \mathrm{kA}$ based on past experience with similar horns used in several neutrino experiments at the BNL AGS [16].

\section{Target region layout and geometry}

The NuMI beam target region, which is shown with a yellow background in Figure 10 , is $53 \mathrm{~m}$ long, $6 \mathrm{~m}$ wide, and $6 \mathrm{~m}$ in height. The NuMI GEANT simulation includes iron and concrete blocks used for shielding in the target and horn region, $1.2 \mathrm{~m}$ of concrete shielding around the decay pipe, and the concrete blocks used as the hadron absorber at the end of the decay pipe. The target protection baffle, also made of graphite, is simulated using GEANT. The GEANT simulation of the full NuMI target region, including shielding, is shown in Figure 13. The red lines indicate the path of charged hadrons (including the proton beam) through the simulation for a $120 \mathrm{GeV}$ proton beam.

For the preliminary implementation of the WBLE target region simulation we have used a $6 \mathrm{~m}$ wide and $6 \mathrm{~m}$ high region. We varied the length of the target region depending on the length of the decay volume simulated. For a decay pipe of $677 \mathrm{~m}$ length (like NuMI) we used a $49 \mathrm{~m}$ long target region, for decay pipes of $180 \mathrm{~m}$ and $380 \mathrm{~m}$ in length, we used a shorter target region of $19 \mathrm{~m}$ in length, effectively moving the downstream end of the WBLE horn 2 as close as possible to the decay pipe window. We have included blocks of iron shielding in the target area. The shielding around the WBLE decay pipe was increased in thickness to $2.5 \mathrm{~m}$ to accommodate the more powerful beam expected.

The GEANT simulation of particles from a $120 \mathrm{GeV}$ beam incident on the WBLE target 
TABLE II: Horn parameters: NuMI and WBLE

\begin{tabular}{lcc}
\hline Component & NuMI & WBLE \\
\hline & Focusing magnetic horn 1 & \\
Shape: & Double parabolic & AGS geometry \\
Conductor: & Al & Al \\
Inner conductor thickness: & $2 \mathrm{~mm}$ min & $2.5 \mathrm{~mm}$ \\
& $4.5 \mathrm{~mm}$ (max at neck) & \\
Outer conductor : & constant & maximum \\
& & \\
Minimum aperture & $9 \mathrm{~mm}$ radius & $7 \mathrm{~mm}$ radius \\
field-free neck: & $3.3 \mathrm{~m}$ & $2.19 \mathrm{~m}$ \\
Length: & $\leq 200 \mathrm{kA}$ & $\leq 250 \mathrm{kA}$ \\
Current: & Water spray & Water spray \\
Cooling: & &
\end{tabular}

\section{Focusing magnetic horn 2}

Shape:

Conductor:

Inner conductor thickness:

Outer conductor :

Double parabolic

$\mathrm{Al}$

$3 \mathrm{~mm} \min$

$5 \mathrm{~mm} \max$
AGS geometry

$\mathrm{Al}$

$1.5 \mathrm{~mm}$

29.13 inch ID 29.82 inch OD 35.43 inch ID 37.26 inch OD
constant
maximum

Minimum aperture

field-free neck:

$3.9 \mathrm{~cm}$ radius

$5.8 \mathrm{~cm}$ radius

Length:

$$
3.58 \mathrm{~m}
$$

$1.57 \mathrm{~m}$

Current:

$\leq 200 \mathrm{kA}$

$\leq 250 \mathrm{kA}$

Distance from $\mathrm{H} 1$ upstream end

$10 \mathrm{~m}$

$10 \mathrm{~m}$

Cooling:

Water spray

Water spray 

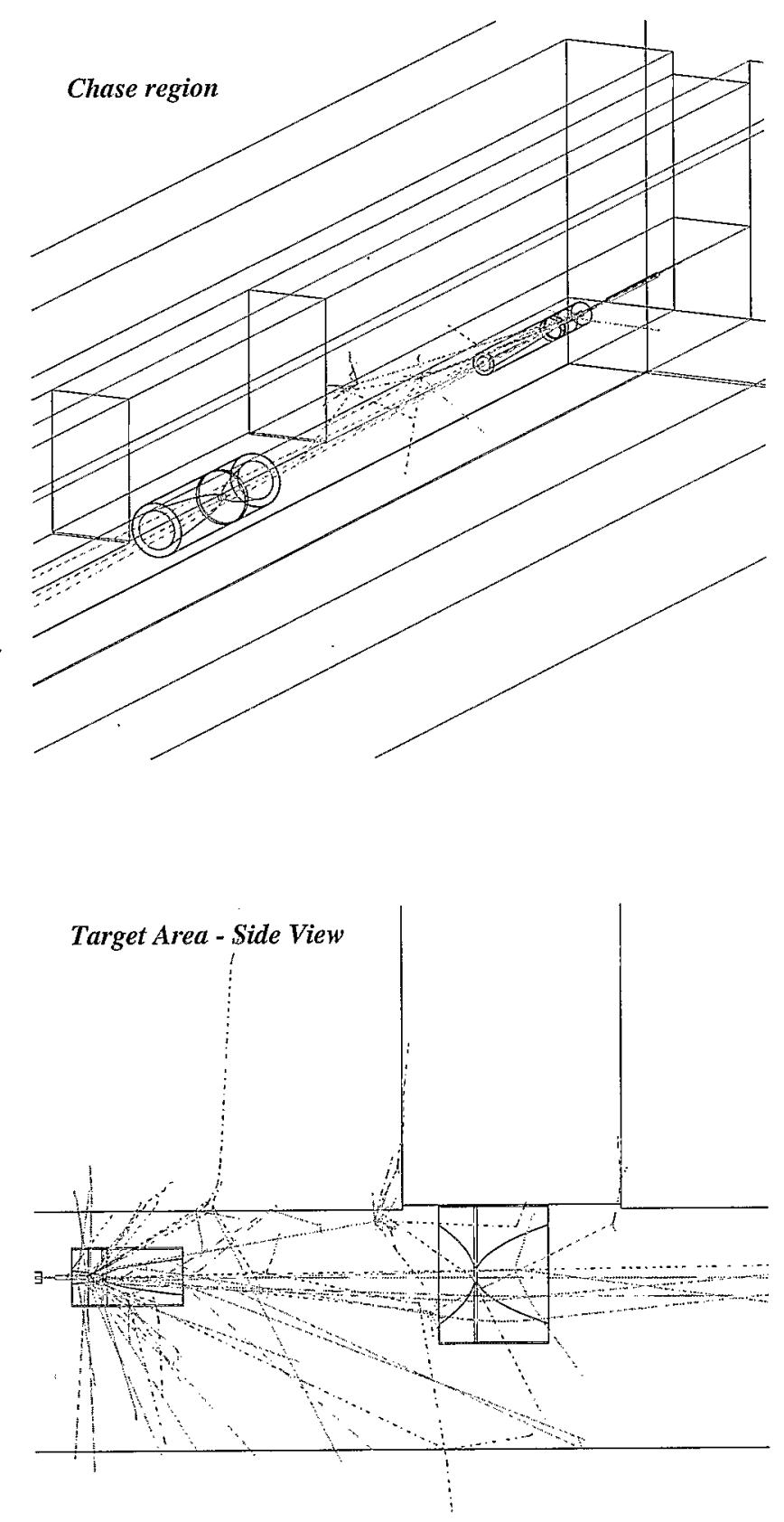

FIG. 13: GEANT simulation of the NuMI target and chase region with $120 \mathrm{GeV} \mathrm{p}+$. The ratio of horizontal to vertical scales in the top figure is $1: 1$ and $1: 5$ in the bottom figure. 


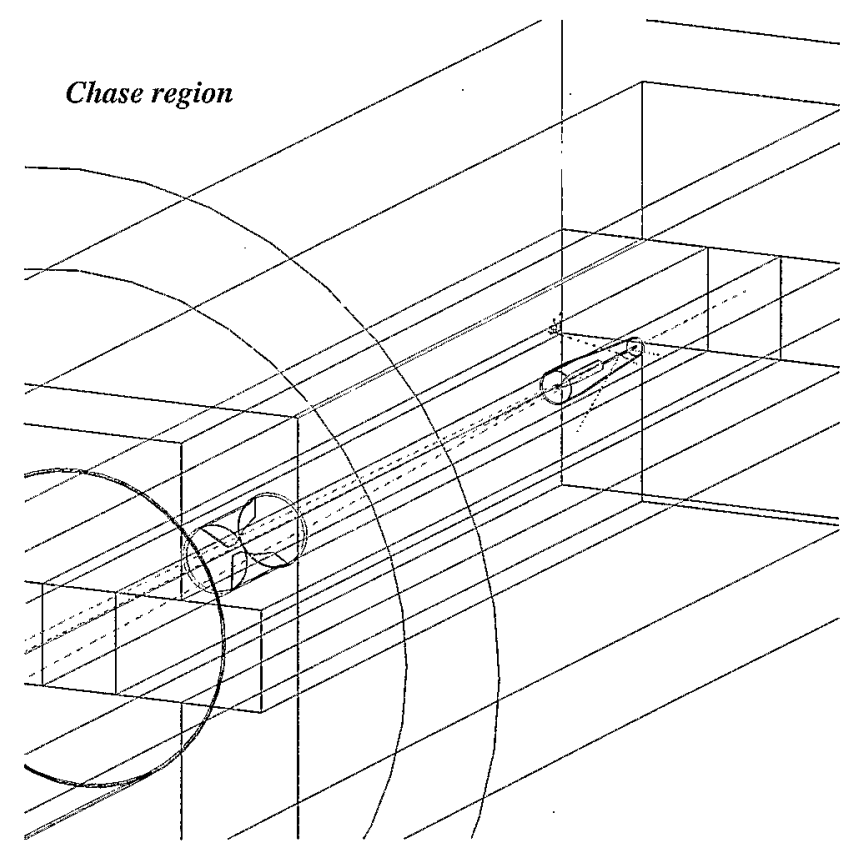

Target Area - Side View
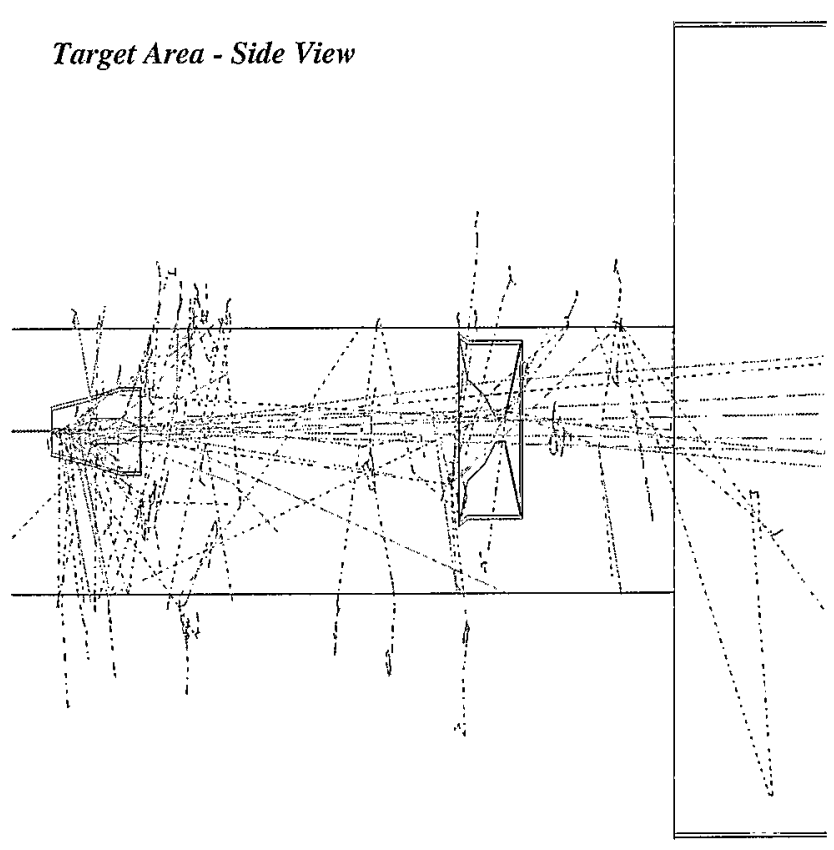

FIG. 14: GEANT simulation of the WBLE target and chase region with $120 \mathrm{GeV}$ p+. The chase region used for this simulation is $19 \mathrm{~m}$ in length. The ratio of horizontal to vertical scales in the top figure is $1: 1$ and 1:5 in the bottom figure. 
and horns in the target region is shown in Figure 14. The target region design for the shorter decay pipes of 180 and $380 \mathrm{~m}$ in length is shown.

\section{Comparative studies with NuMI and AGS simulations}

The WBLE target and horn design in the current FLUKA '05/GEANT simulation was optimized for the $28 \mathrm{GeV}$ AGS beam. To determine how well the design functions at the higher proton beam energies expected from the MI, we compare the neutrino flux at $1 \mathrm{~km}$ from the target produced by the NuMI target and horn design to that produced from the WBLE target and horns. In this simulation we have kept the decay tunnel and target region geometries identical for the NuMI and WBLE simulation - only the targets and horns have been changed. The proton beam energy is set at $120 \mathrm{GeV}$. We used two settings for the horn currents for each beam-line: $0 \mathrm{kA}$ and $185 \mathrm{kA}$. The results of the comparative study of the NuMI beam-line in its standard operating mode to that of the WBLE beam-line target/horn design is shown in Figure 15.

We observe the following:

- The high energy tails from unfocused hadrons in both the NuMI and WBLE simulations are almost identical in magnitude for energies $>10 \mathrm{GeV}$. This gives us confidence that the normalization of the absolute neutrino rates obtained from the WBLE target and horn simulation is reliable, based on the measurements done with the MINOS near detector.

- The shape of the spectrum in the region 0 to $10 \mathrm{GeV}$ is much broader in the WBLE simulation as compared to NuMI with more neutrinos produced in the sub GeV range. This validates that the WBLE target and horn design does indeed produce a wide-band beam with enhanced flux in the low energy region as expected.

- The integrated neutrino flux at $1 \mathrm{~km}$ from the target in the region $0-10 \mathrm{GeV}$ from the NuMI and WBLE simulation is $3.074 \times 10^{-4} \nu / \mathrm{m}^{2} / \mathrm{POT}$ and $3.015 \times 10^{-4} \nu / \mathrm{m}^{2} / \mathrm{POT}$ 


\section{NUMI LE-10 vs WBLE spectra}

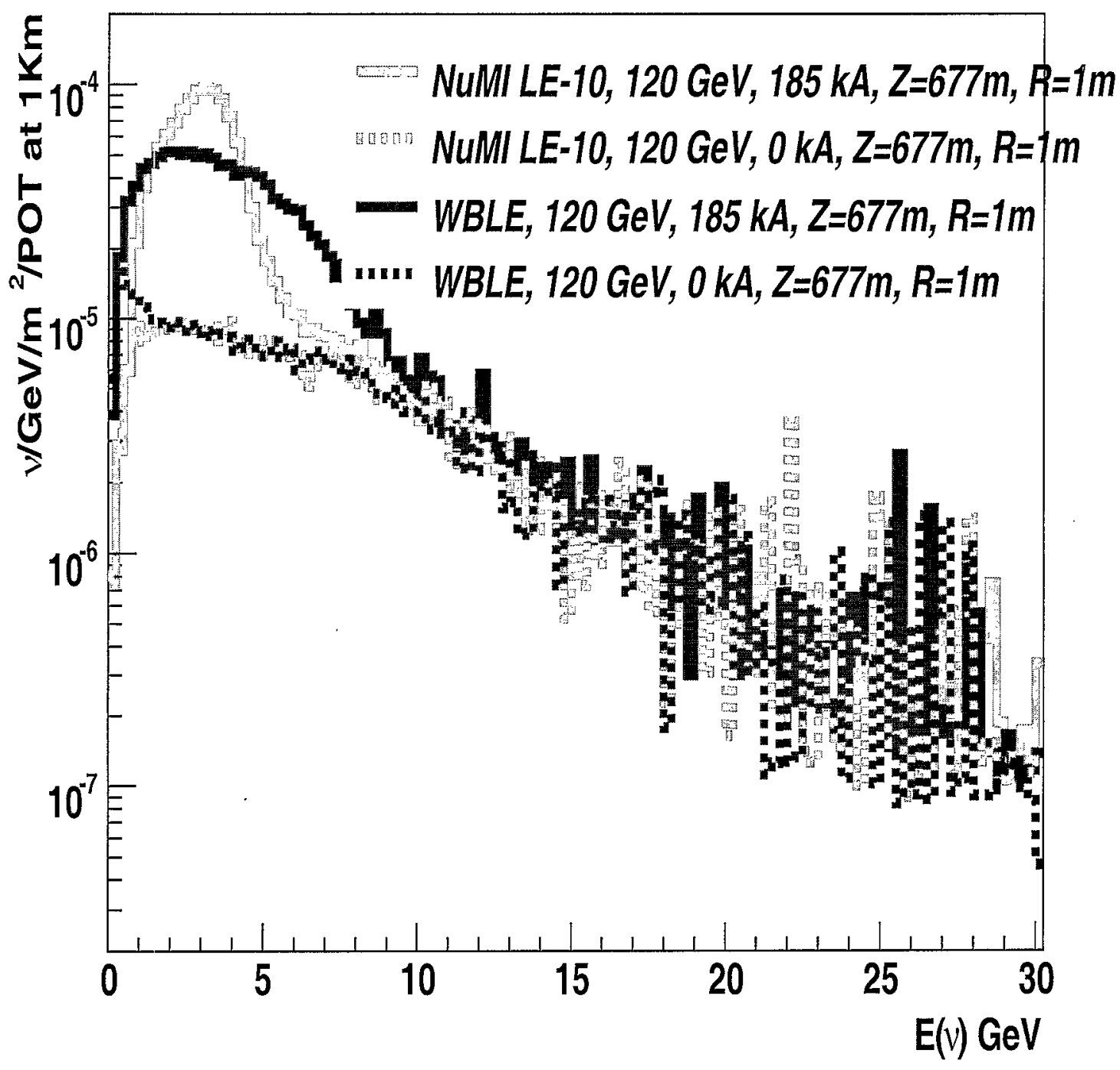

FIG. 15: The neutrino flux from the WBLE horn and target design as compared to the NuMI neutrino flux. The beam energy of $120 \mathrm{GeV}$ and the target area and decay tunnel geometries are identical in both simulations. The results are shown for the horn currents set to 0 and $185 \mathrm{kA}$.

respectively. This indicates that, although the WBLE horn and target designs have not been optimized for the higher energy beam, the integrated neutrino flux is only a few $\%$ different from that produced by the NuMI beam-line when the same beam-line geometry is used. 
- When the horns are turned off the absolute neutrino flux from the WBLE and NuMI in the $1-10 \mathrm{GeV}$ range is almost identical. This is a validation that the hadron-production from the WBLE target simulation implemented in FLUKA'05 is in good agreement with the expectations from NuMI. There is an enhancement of the WBLE flux in the sub-GeV range compared to NuMI that needs further investigation, but could be the result of using a shorter, thicker target.

- The ratio of the WBLE flux at $<5 \mathrm{GeV}$ to that at $>5 \mathrm{GeV}$ for this beam energy and decay tunnel design is only $2: 1$. This implies that the NuMI beam energy and decay tunnel design is far from optimal for the VLBNO program envisioned in [1] and [2]. Therefore, further optimization of the WBLE beam-line design is needed.

The current estimates of the physics sensitivities of a wide band on-axis long baseline experiment [1] [2] used a simulation of $28 \mathrm{GeV}$ beam from the BNL AGS with the same horn design as described previously. We compare the neutrino flux from these recent simulations with the previous results in Figure 16. The decay pipe geometry in the new WBLE simulation is set to be $2 \mathrm{~m}$ in radius and $180 \mathrm{~m}$ long to match the AGS study and the horn currents are set to $250 \mathrm{kA}$. Given that the target simulation used in the AGS study was based on an older version of GEANT and the current WBLE target simulation uses FLUKA '05, these simulations are expected to have different hadro-production spectra. It should also be noted that measurements using the AGS beam have concluded that FLUKA '05 is in better agreement with hadro-production data at the AGS [14] compared to the GEANT simulations. There are some other minor discrepancies between the two simulations that have not yet been addressed: the distance between horn 1 and horn 2 is $8.3 \mathrm{~m}$ in the AGS beam whereas in the the WBLE simulation the distance between the two horns is $10 \mathrm{~m}$. There are also some minor residual discrepancies in the horn conductor thicknesses between the two studies. The results shown in Figure 16 demonstrate that the peak energy and the flux at the peak are in good agreement in both simulations. The integrated $\nu_{\mu}$ flux in the region of $0-5 \mathrm{GeV}$ for the AGS and WBLE simulations is also in good agreement being 
WBLE vs AGS beam spectra

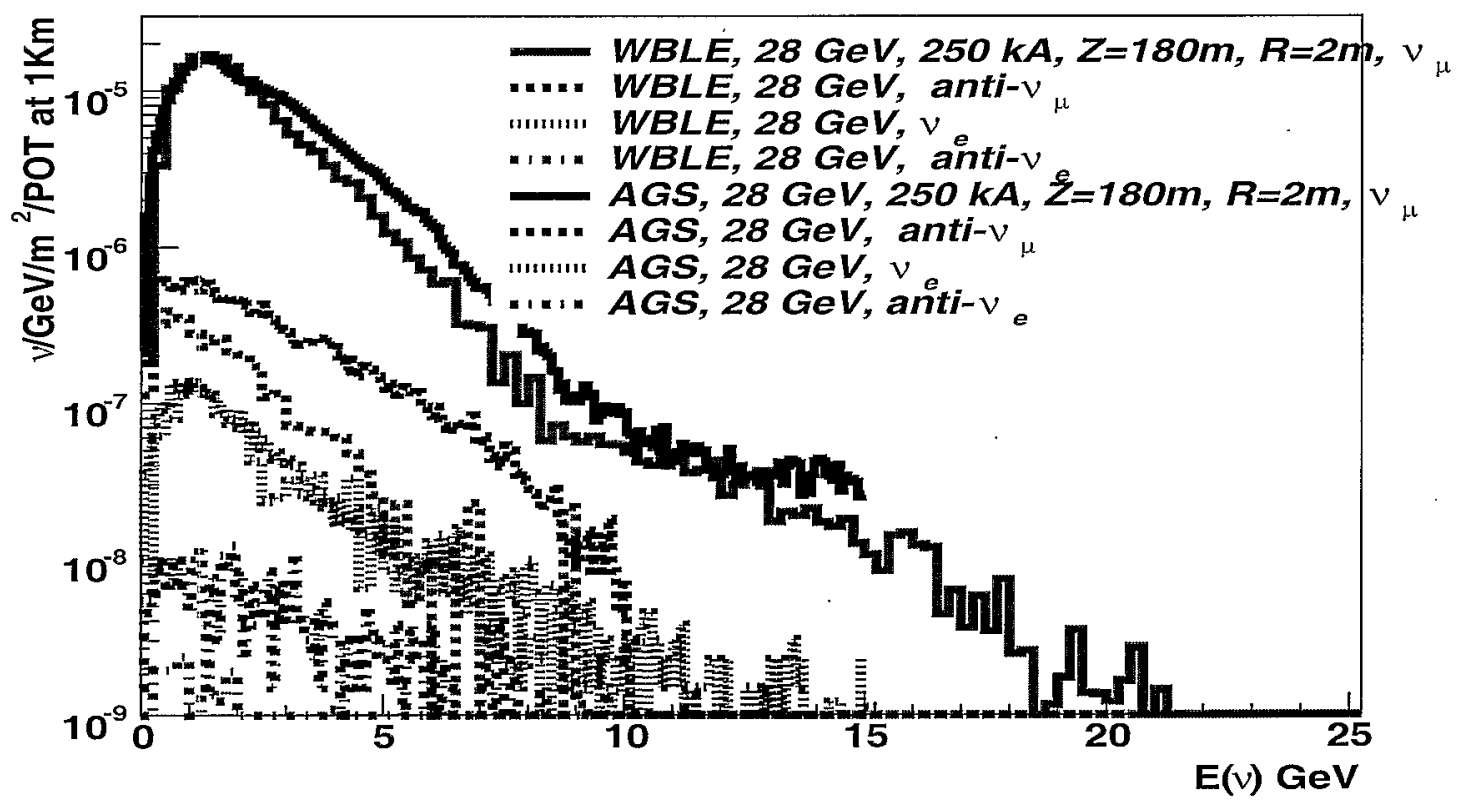

WBLE vs AGS beam spectra

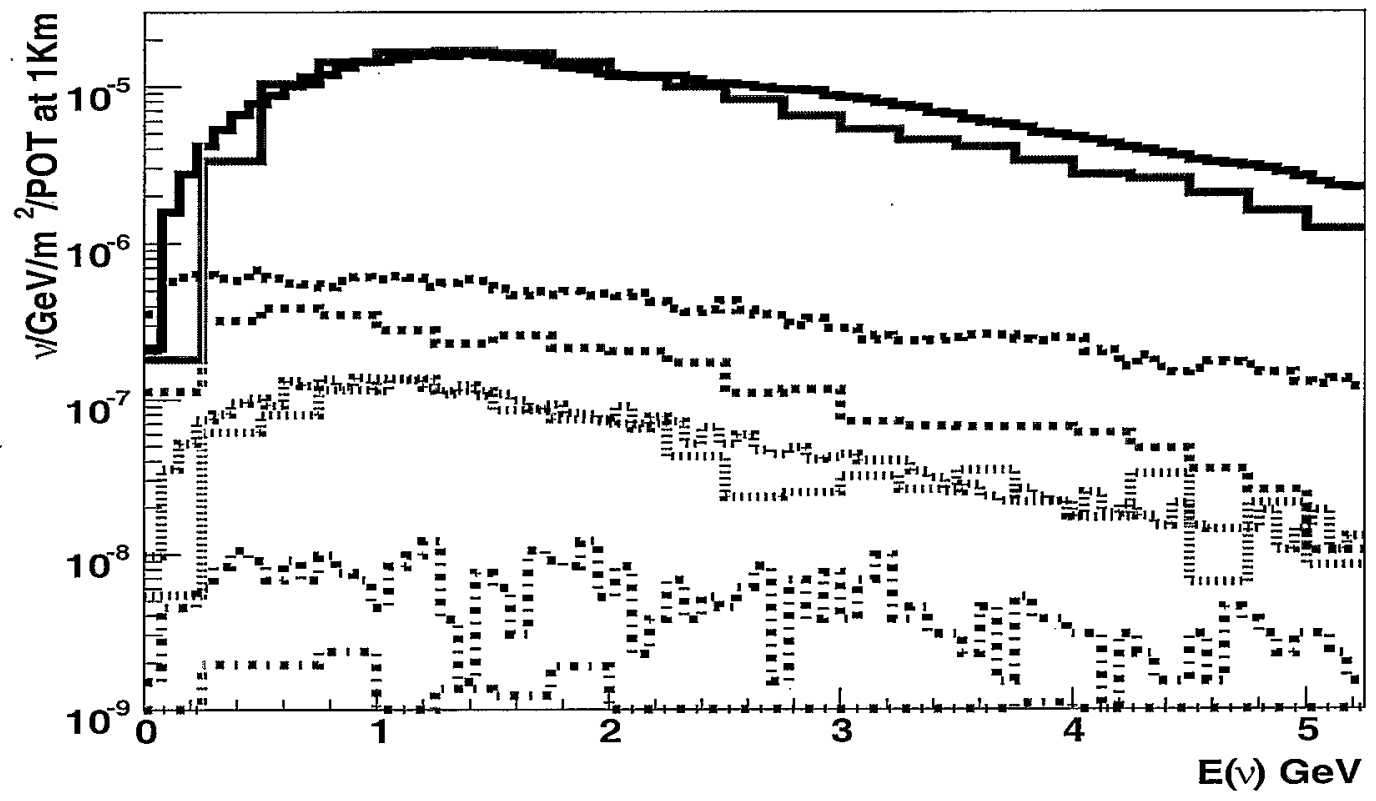

FIG. 16: Comparison of the WBLE simulation for a $28 \mathrm{GeV}$ beam with the previous results obtained in the BNL study

$4.30 \times 10^{-5} \nu / \mathrm{m}^{2} /$ POT and $3.90 \times 10^{-5} \nu / \mathrm{m}^{2} /$ POT respectively. The spectrum from the two simulations at energies $>5 \mathrm{GeV}$ is different, with the WBLE simulation predicting much 
lower fluxes at high energy. In addition, the anti-neutrino contamination is a factor of $\times 2.6$ lower in the WBLE simulation. The $\nu_{e}$ contamination spectrum and flux is almost identical. in both simulations. 


\section{OPTIMIZATION OF THE FERMILAB WBLE BEAM SPECTRUM}

In the previous section we simulated various WBLE beamline configurations and established that our simulations should be reasonably accurate based on comparisons with the FNAL NuMI simulation. We also demonstrated that the energy of the NuMI beam energy $(120 \mathrm{GeV})$ and the NuMI decay tunnel design are not optimal for the next generation VLBNO program using the WBLE horn and target design. Current siting restrictions at Fermilab for a beam-line to DUSEL limit the decay pipe length to $<400 \mathrm{~m}$ and the radius to $<2 \mathrm{~m}$. In Figure 17, we compare the WBLE neutrino flux from a $60 \mathrm{GeV}$ MI beam with the NuMI decay pipe geometry (1m radius $677 \mathrm{~m}$ length) and a decay pipe that is $380 \mathrm{~m}$ in length and $2 \mathrm{~m}$ in radius. We find that the wider-shorter decay pipe geometry produces a larger flux in the desired region of $0-5 \mathrm{GeV}$, a smaller flux in the region $5-15 \mathrm{GeV}$ and similar flux at $>15 \mathrm{GeV}$ [21]. We varied the MI proton beam energy in the WBLE simulation from 28 to $120 \mathrm{GeV}$ coupled with a decay pipe of $380 \mathrm{~m}$ in length and $2 \mathrm{~m}$ in radius. We also simulated a $28 \mathrm{GeV}$ WBLE beam using a decay pipe of $180 \mathrm{~m}$ length and $2 \mathrm{~m}$ radius.

The different neutrino fluxes from the WBLE beam-line design using different beam energies and decay tunnel geometries are shown in Figure 18. The properties of the neutrino flux spectrum from some of the different WBLE beam configurations studied are summarized in Table IV.

We find that at a beam energy of $120 \mathrm{GeV}$ with the decay pipe of $380 \mathrm{~m}$ in length and $2 \mathrm{~m}$ in radius, the peak flux at $1 \mathrm{~km}$ is almost comparable with NuMI but the ratio of the flux in the region $<5 \mathrm{GeV}$ compared to the flux at $>5 \mathrm{GeV}$ is still only 2.5:1. This indicates that, even with the shorter decay tunnel, at $120 \mathrm{GeV}$ the neutral current backgrounds from the high-energy tails will still be substantial. The relative size of the high energy tail greatly improves as the energy is lowered from $120 \mathrm{GeV}$ to $28 \mathrm{GeV}$, but the peak flux ( per protonon-target) decreases by a factor of four, and the peak energy moves further away from the 1st oscillation maxima of $2.4 \mathrm{GeV}$. The possibility of using a beam plug between the two WBLE horns to eliminate the high energy tail at higher beam energies while preserving most 


\section{WBLE, $60 \mathrm{GeV}$ beam with different decay volumes}

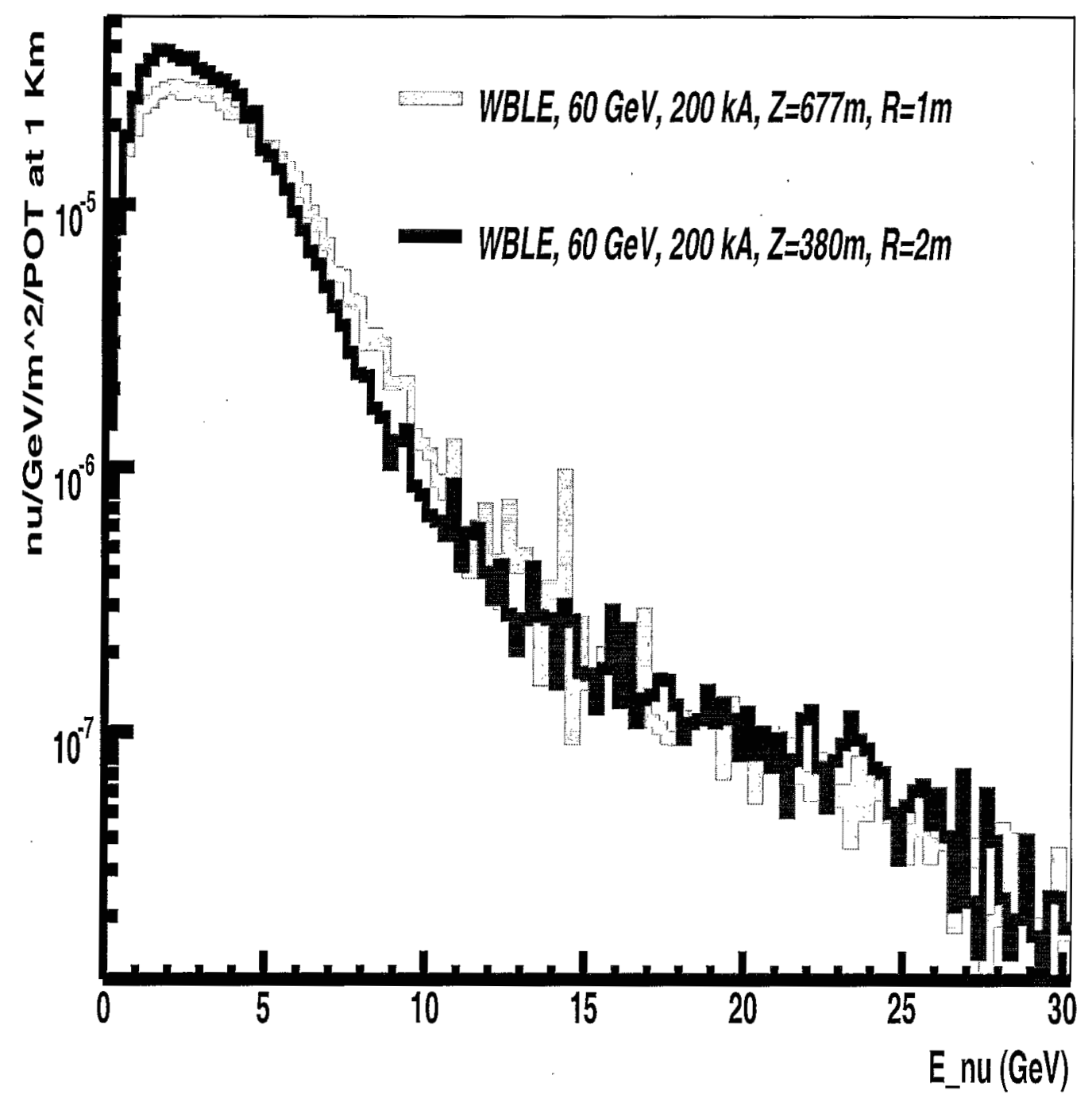

FIG. 17: WBLE neutrino fluxes at $1 \mathrm{~km}$ using $60 \mathrm{GeV}$ and different decay volume geometries. The decay pipe is cylindrical where $R$ is the decay pipe radius and $Z$ indicates the decay pipe length.

of the flux in the low energy region will be studied. Based on these preliminary results of the simulation of a WBLE beam spectrum and event rates from the Fermilab MI beam, we recommend further consideration of the physics sensitivities of the VLBNO program using the 60 and $40 \mathrm{GeV}$ beam configurations shown in Figures 19, and 20 respectively. 


\section{WBLE beam, different energies, decay tunnels}

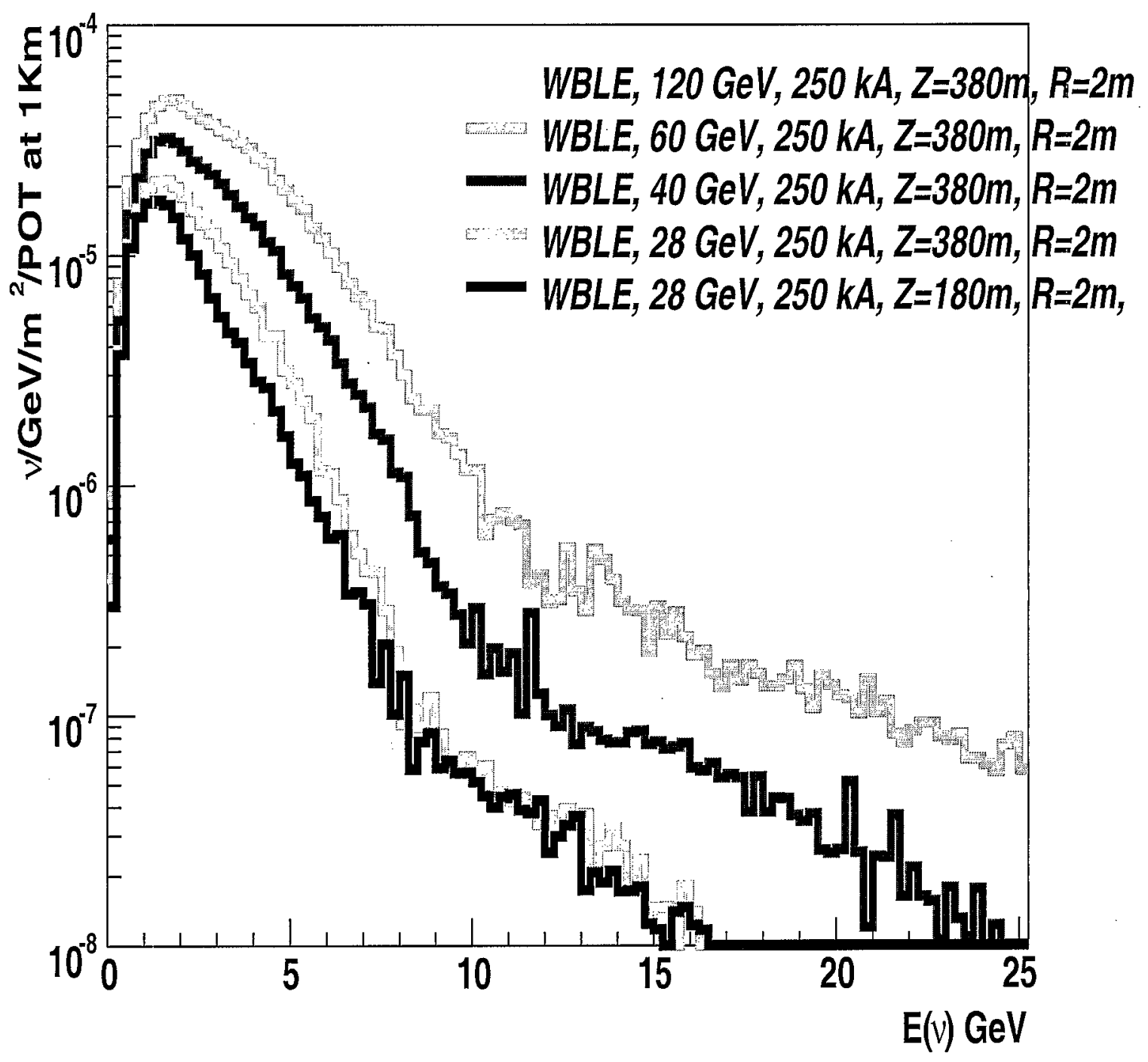

FIG. 18: WBLE neutrino fluxes at $1 \mathrm{~km}$ using different energies and decay volume configurations. $R$ is the decay tunnel radius and $Z$ indicates the decay tunnel length. 
TABLE III: Properties of different WBLE designs as compared to the NuMI and BNL-AGS beams. The first column indicates the beam design used, the decay pipe geometry where $\mathrm{R}$ is the radius and $\mathrm{Z}$ is the length, and the horn current. The second column indicates the neutrino energy at which the flux is maximal and the value of that flux at $1 \mathrm{~km}$ from the target. The $3 \mathrm{rd}$ and $4 \mathrm{th}$ columns indicate the neutrino energy at which the flux has fallen to $50 \%$ and $10 \%$ of the peak flux respectively.

\begin{tabular}{|c|c|c|c|c|c|}
\hline $\begin{array}{c}\text { Beam } \\
\text { Scenario }\end{array}$ & $\begin{array}{c}\text { Peak } E \\
\text { (Flux at } 1 \mathrm{~km})\end{array}$ & $\begin{array}{c}E, 50 \% \\
\max \text { flux }\end{array}$ & $\begin{array}{l}E, 10 \% \\
\max \text { flux }\end{array}$ & $\begin{array}{c}\text { Flux ratio } \\
\frac{<5 \mathrm{GeV}}{>5 \mathrm{GeV}}\end{array}$ & $\begin{array}{l}\text { Purity } \\
\text { overall }\end{array}$ \\
\hline & $\nu /\left(\mathrm{GeV} \cdot \mathrm{m}^{2} \cdot \mathrm{pot}\right)$ & $\mathrm{GeV}$ & $\mathrm{GeV}$ & & \\
\hline $\begin{array}{l}\text { NuMI LE-10 } \\
\begin{array}{l}\mathrm{Z}=677, \mathrm{R}=1 \mathrm{~m} \\
185 \mathrm{kA}\end{array}\end{array}$ & $\begin{array}{c}3.1 \pm 0.13 \mathrm{GeV} \\
\left(9.7 \times 10^{-5}\right)\end{array}$ & $4.1 \pm 0.13$ & $6.1 \pm 0.13$ & 4 & $\begin{array}{c}89 \% \nu_{\mu} \\
1 \% \nu_{e}\end{array}$ \\
\hline $\begin{array}{l}\text { WBLE } 120 \mathrm{GeV} \\
\begin{array}{c}\mathrm{Z}=380, \mathrm{R}=2 \mathrm{~m} \\
250 \mathrm{kA}\end{array}\end{array}$ & $\begin{array}{c}1.9 \\
\left(8.1 \times 10^{-5}\right)\end{array}$ & 5.5 & 8.6 & 2.5 & $\begin{array}{l}93 \% \nu_{\mu} \\
0.85 \% \nu_{e}\end{array}$ \\
\hline $\begin{array}{l}\text { WBLE } 60 \mathrm{GeV} \\
\mathrm{Z}=380, \mathrm{R}=2 \mathrm{~m} \\
250 \mathrm{kA}\end{array}$ & $\begin{array}{c}1.9 \\
\left(4.7 \times 10^{-5}\right)\end{array}$ & 4.6 & 7.3 & 5 & $\begin{array}{l}96 \% \nu_{\mu} \\
0.86 \% \nu_{e}\end{array}$ \\
\hline $\begin{array}{l}\text { WBLE } 40 \mathrm{GeV} \\
\mathrm{Z}=380, \mathrm{R}=2 \mathrm{~m} \\
250 \mathrm{kA}\end{array}$ & $\begin{array}{c}1.7 \\
\left(3.2 \times 10^{-5}\right)\end{array}$ & 3.6 & 6.3 & 8 & $\begin{array}{l}96 \% \nu_{\mu} \\
0.99 \% \nu_{e}\end{array}$ \\
\hline $\begin{array}{l}\text { WBLE } 28 \mathrm{GeV} \\
\begin{array}{c}\mathrm{Z}=380, \mathrm{R}=2 \mathrm{~m} \\
250 \mathrm{kA}\end{array}\end{array}$ & $\begin{array}{c}1.6 \\
\left(2.1 \times 10^{-5}\right)\end{array}$ & 3.2 & 5.4 & 16 & $\begin{array}{l}97 \% \nu_{\mu} \\
0.97 \% \nu_{e}\end{array}$ \\
\hline $\begin{array}{l}\text { WBLE } 28 \mathrm{GeV} \\
\begin{array}{l}\mathrm{Z}=180, \mathrm{R}=2 \mathrm{~m} \\
250 \mathrm{kA}\end{array}\end{array}$ & $\begin{array}{c}1.3 \\
\left(1.8 \times 10^{-5}\right)\end{array}$ & 2.6 & 5.1 & 21 & $\begin{array}{l}97 \% \nu_{\mu} \\
0.67 \% \nu_{e}\end{array}$ \\
\hline $\begin{array}{c}\text { AGS } 28 \mathrm{GeV} \\
\mathrm{Z}=180, \mathrm{R}=2 \mathrm{~m} \\
250 \mathrm{kA}\end{array}$ & $\begin{array}{l}1.39 \pm 0.04 \\
\left(1.6 \times 10^{-5}\right)\end{array}$ & 3.11 & 5.81 & 12 & $\begin{array}{l}95 \% \nu_{\mu} \\
0.7 \% \nu_{e}\end{array}$ \\
\hline
\end{tabular}




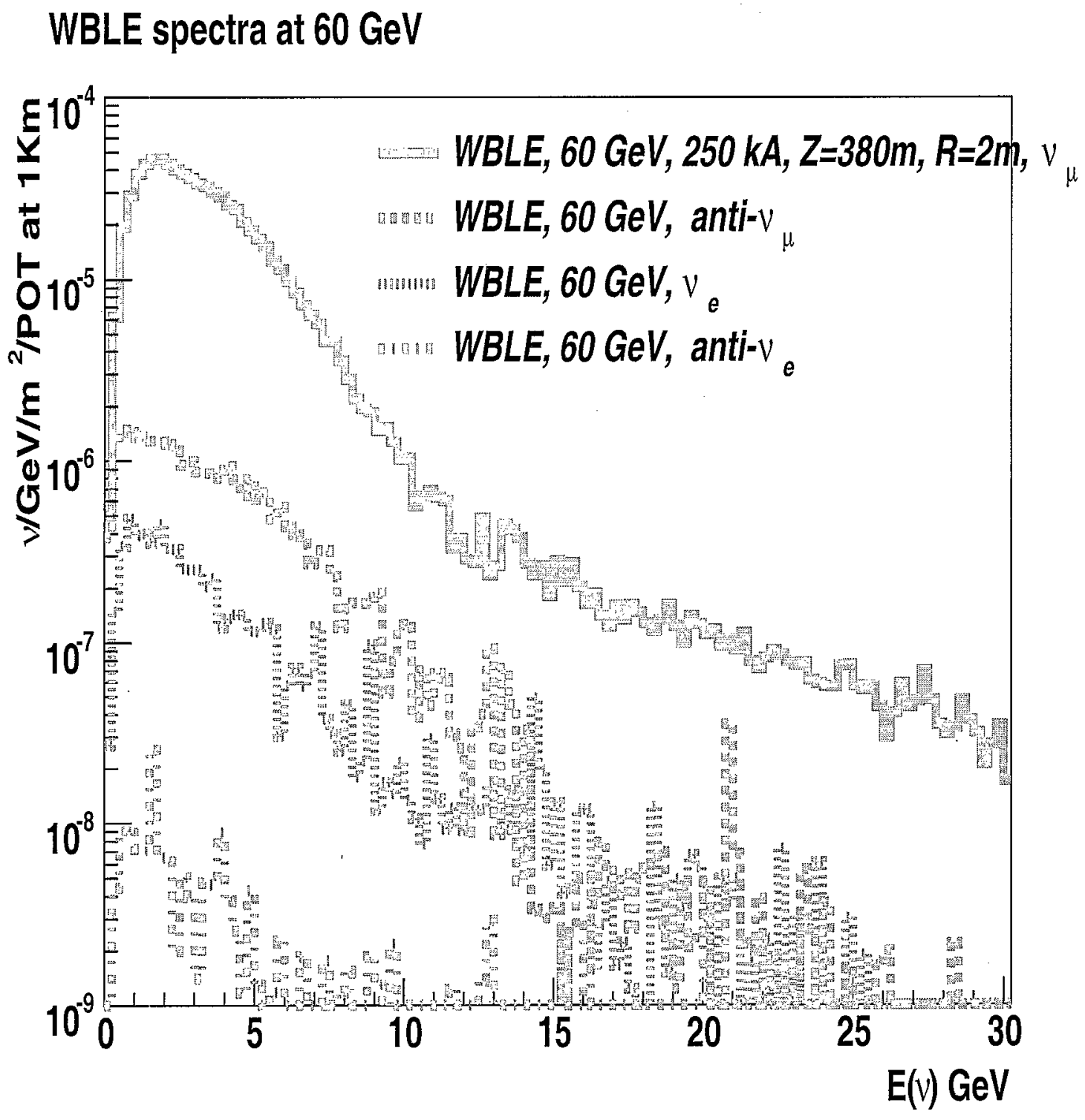

FIG. 19: WBLE $60 \mathrm{GeV}$ beam spectra and flux components 


\section{WBLE spectra at $40 \mathrm{GeV}$}

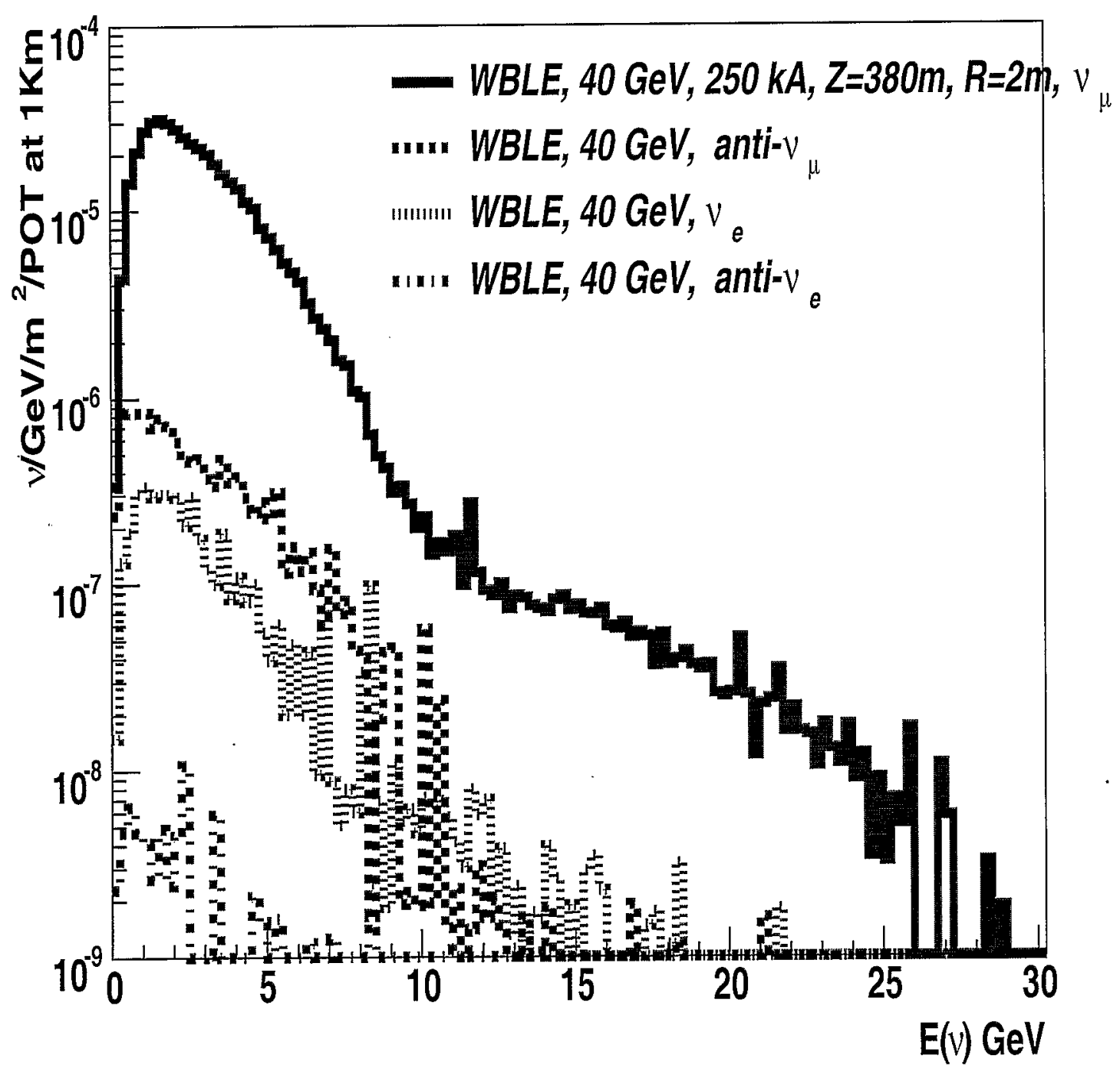

FIG. 20: WBLE $40 \mathrm{GeV}$ beam spectra and flux components 


\section{EVENT RATES AT THE FAR DETECTOR}

In this section we present some preliminary estimates of the observed $\mathrm{CC}$ and QE interaction rates from different beam configurations at a far detector located in The Homestake Mine, $1297 \mathrm{~km}$ from the target.

Assuming the detector has perfect energy resolution and efficiency, we compute the event rates thus:

$$
\text { Event rate }=\sigma_{C C, Q E} \times F
$$

where $\sigma_{C C, Q E}$ are the total charged-current and quasi-elastic charged-current cross-sections respectively in $m^{2}$ per kiloton of detector and $F$ are the neutrino fluxes from the various beam configurations calculated at a distance of $1297 \mathrm{~km}$ from the target. Different types of detector target material have different populations of neutrons and protons and slightly different values of $\sigma_{C C, Q E}$ per kiloton of detector. The total CC and quasi-elastic (QE) interaction rates per nucleon used are shown in Figure 21.

The total CC interaction rates, the rates at the oscillation maxima and the rates in the region $<5 \mathrm{GeV}$ are summarized for different beam configurations in Table IV. The rates are given in units of events per GeV. $10^{20}$ protons-on-target. $\mathrm{m}^{2}$. In table $\mathrm{V}$, we also present the event rates in units of MW.kT.107 seconds. The following formula is used to convert numbers of protons to MW. $10^{7}$ seconds:

$$
\text { Number of protons }\left(10^{20}\right)=\frac{1000 \times \text { Beam Power }(\mathrm{MW}) \text {.time }\left(10^{7} \mathrm{~s}\right)}{1.602 E_{p}(\mathrm{GeV})}
$$

where $E_{p}$ is the proton beam energy.

Current plans for the VLBNO program indicate that Water Cerenkov detector technology is one of the candidates for the far detector, therefore the CC quasi-elastic event rates in Table $\mathrm{V}$ are given in units of kiloton of water.

We find that the $40 \mathrm{GeV}$ MI WBLE beam is a close match in the ratio of low energy relative to high energy neutrino flux to the AGS beam, but with $50 \%$ larger integrated CC rate at the far detector for the same beam power. The $60 \mathrm{GeV}$ MI WBLE beam has a factor 

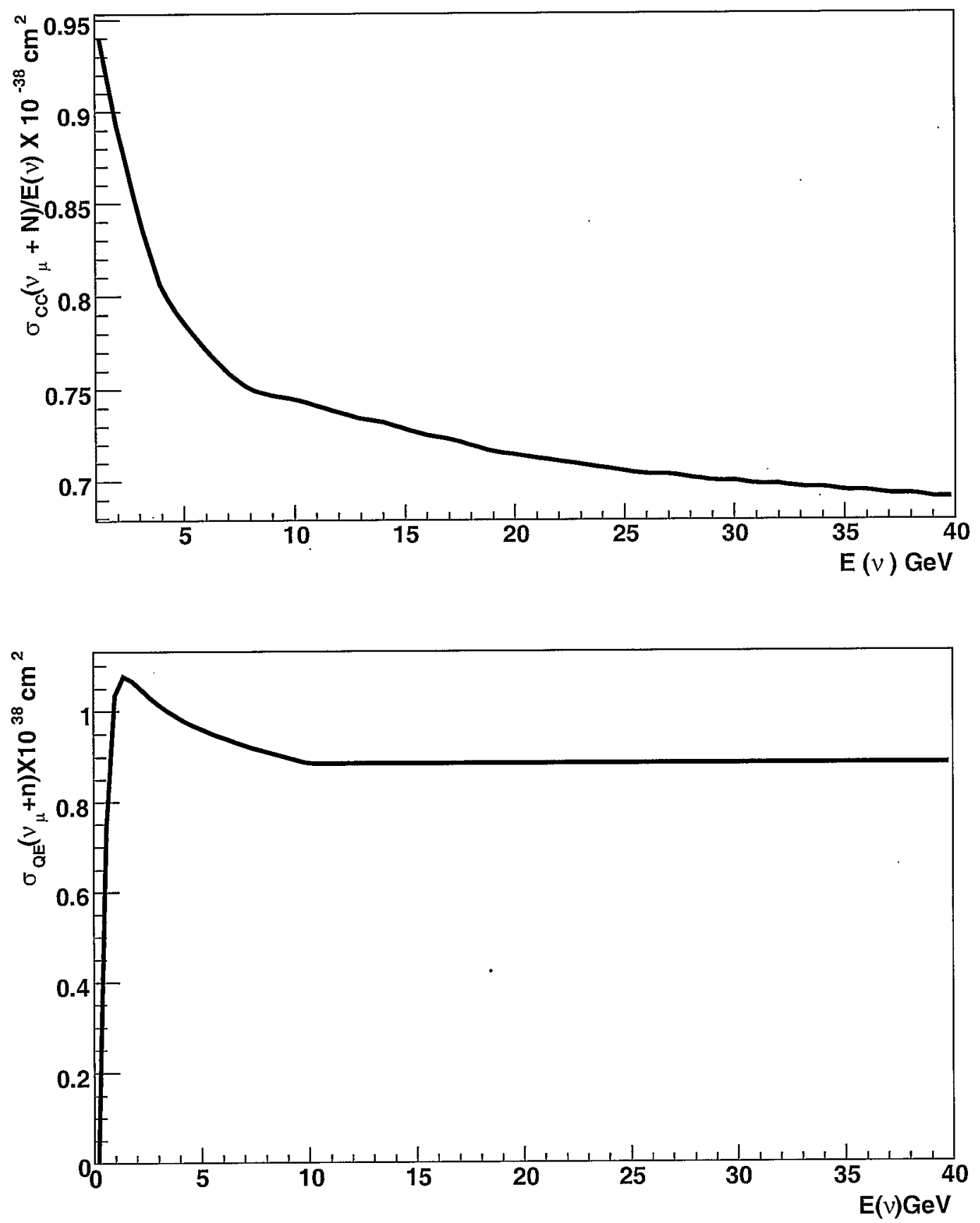

FIG. 21: Total charged-current (top) and quasi-elastic (bottom) interaction cross-sections per nucleon. The total charged-current cross-section per nucleon is the average for an Fe56 nucleus: $\sigma_{C C}\left(\nu_{\mu}+N\right)=\left(26 \sigma_{C C}\left(\nu_{\mu}+p\right)+30 \sigma\left(\nu_{\mu}+n\right)\right) / 56$

of 2 higher ratio of the neutrino flux at $>5 \mathrm{GeV}$ to $<5 \mathrm{GeV}$ as compared to the AGS beam 
TABLE IV: Charged-current (CC) rates for Fermilab-HOMESTAKE using different WBLE designs. For comparison the numbers for NuMI LE-10 configuration and the AGS beam are also noted. This is for a horn current of $250 \mathrm{kA}$ for the WBLE beam. The energy at which the CC rate peaks, the CC rate at the peak energy, the first and second oscillation maxima and the rates at the oscillation maxima are indicated in columns 2,3 and 4 . In addition, the integrated CC interaction rate and the integrated rate in the $0-5 \mathrm{GeV}$ region are shown in the last column.

\begin{tabular}{|c|c|c|c|c|}
\hline $\begin{array}{c}\text { Beam } \\
\text { Scenario }\end{array}$ & $\begin{array}{c}\text { Peak } E \\
\left(\mathrm{CC} \text { rate, } R_{p}\right) \\
/\left(\mathrm{GeV} \cdot 10^{20} \text { pot.kT }\right)\end{array}$ & $\begin{array}{c}\text { 1st osc max } \mathrm{E} \\
\left(\mathrm{CC} \text { rate, } R_{1}\right) \\
/\left(\mathrm{GeV} .10^{20} \text { pot.kT }\right)\end{array}$ & $\begin{array}{l}\text { 2nd osc max } \mathrm{E} \\
\left(\mathrm{CC} \text { rate } R_{2}\right) \\
\left(\mathrm{GeV} \cdot 10^{20} \text { pot.kT) }\right.\end{array}$ & $\begin{array}{c}\text { Total CC rate } R_{\text {total }} \\
\qquad \begin{array}{c}\left(R_{<5}<5 \mathrm{GeV}\right) \\
/\left(10^{20} \text { pot.kT }\right)\end{array}\end{array}$ \\
\hline \multicolumn{5}{|c|}{ At a distance of $735 \mathrm{~km}$} \\
\hline $\begin{array}{l}\text { NuMI LE-10 } \\
\mathrm{Z}=677, \mathrm{R}=1 \mathrm{~m}\end{array}$ & $\begin{array}{c}E_{p}=3.4 \pm 0.125 \mathrm{GeV} \\
\left(R_{p}=18\right)\end{array}$ & $\begin{array}{c}E_{1}=1.5 \mathrm{GeV} \\
\left(R_{1}=6.1\right)\end{array}$ & $\begin{array}{l}E_{2}=0.5 \mathrm{GeV} \\
\left(R_{2}=0.123\right)\end{array}$ & $\begin{array}{l}R_{\text {total }}=82 \\
\left(R_{<5}=49\right) \\
\end{array}$ \\
\hline \multicolumn{5}{|c|}{ At a distance of $1297 \mathrm{~km}$} \\
\hline $\begin{array}{l}\text { WBLE } 120 \mathrm{GeV} \\
\mathrm{Z}=380, \mathrm{R}=2 \mathrm{~m}\end{array}$ & $\begin{array}{c}E_{p}=4.6 \mathrm{GeV} \\
\left(R_{p}=5.3\right)\end{array}$ & $\begin{array}{c}E_{1}=2.4 \mathrm{GeV} \\
\left(R_{1}=4.3\right)\end{array}$ & $\begin{array}{c}E_{2}=0.8 \mathrm{GeV} \\
\left(R_{2}=1.1\right)\end{array}$ & $\begin{array}{l}R_{\text {total }}=44 \\
\left(R_{<5}=19\right)\end{array}$ \\
\hline $\begin{array}{l}\text { WBLE } 60 \mathrm{GeV} \\
\mathrm{Z}=380, \mathrm{R}=2 \mathrm{~m}\end{array}$ & $\begin{array}{c}E_{p}=3.4 \mathrm{GeV} \\
\left(R_{p}=2.4\right)\end{array}$ & $\begin{array}{c}E_{1}=2.4 \mathrm{GeV} \\
\left(R_{1}=2.4\right)\end{array}$ & $\begin{array}{c}E_{2}=0.8 \mathrm{GeV} \\
\left(R_{2}=0.67\right)\end{array}$ & $\begin{array}{l}R_{\text {total }}=16 \\
\left(R_{<5}=9.6\right) \\
\end{array}$ \\
\hline $\begin{array}{l}\text { WBLE } 40 \mathrm{GeV} \\
\mathrm{Z}=380, \mathrm{R}=2 \mathrm{~m}\end{array}$ & $\begin{array}{c}E_{p}=2.1 \mathrm{GeV} \\
\left(R_{p}=1.4\right)\end{array}$ & $\begin{array}{c}E_{1}=2.4 \mathrm{GeV} \\
\left(R_{1}=1.4\right)\end{array}$ & $\begin{array}{c}E_{2}=0.8 \mathrm{GeV} \\
\left(R_{2}=0.49\right)\end{array}$ & $\begin{array}{l}R_{\text {total }}=7.6 \\
\left(R_{<5}=5.6\right)\end{array}$ \\
\hline $\begin{array}{l}\text { WBLE } 28 \mathrm{GeV} \\
\mathrm{Z}=380, \mathrm{R}=2 \mathrm{~m}\end{array}$ & $\begin{array}{c}E_{p}=1.9 \mathrm{GeV} \\
\left(R_{p}=0.95\right)\end{array}$ & $\begin{array}{c}E_{1}=2.4 \mathrm{GeV} \\
\left(R_{1}=0.90\right)\end{array}$ & $\begin{array}{c}E_{2}=0.8 \mathrm{GeV} \\
\left(R_{2}=0.35\right)\end{array}$ & $\begin{array}{l}R_{\text {total }}=3.4 \\
\left(R_{<5}=3.1\right)\end{array}$ \\
\hline $\begin{array}{l}\text { WBLE } 28 \mathrm{GeV} \\
\mathrm{Z}=180, \mathrm{R}=2 \mathrm{~m}\end{array}$ & $\begin{array}{c}E_{p}=1.9 \mathrm{GeV} \\
\left(R_{p}=0.80\right)\end{array}$ & $\begin{array}{c}E_{1}=2.4 \mathrm{GeV} \\
\left(R_{1}=0.68\right)\end{array}$ & $\begin{array}{c}E_{2}=0.8 \mathrm{GeV} \\
\left(R_{2}=0.35\right)\end{array}$ & $\begin{array}{l}R_{\text {total }}=2.6 \\
\left(R_{<5}=2.3\right)\end{array}$ \\
\hline $\begin{array}{l}\text { AGS } 28 \mathrm{GeV} \\
\mathrm{Z}=180, \mathrm{R}=2 \mathrm{~m}\end{array}$ & $\begin{array}{c}E_{p}=2.3 \mathrm{GeV} \\
\left(R_{p}=0.84\right)\end{array}$ & $\begin{array}{c}E_{1}=2.4 \mathrm{GeV} \\
\left(R_{1}=0.81\right)\end{array}$ & $\begin{array}{c}E_{2}=0.8 \mathrm{GeV} \\
\left(R_{2}=0.32\right)\end{array}$ & $\begin{array}{l}R_{\text {total }}=3.5 \\
\left(R_{<5}=2.8\right)\end{array}$ \\
\hline
\end{tabular}

but we gain a factor of 2 increase in integrated CC rate at the far detector for the same beam power. This indicates that it might be possible to achieve the same sensitivity estimated for 
TABLE V: Total Charged and quasi-elastic current $\nu_{\mu}$ interaction rates for Fermilab-HOMESTAKE using different WBLE designs. For comparison the numbers for NuMI and the BNL design are also noted.

\begin{tabular}{|c|c|c|c|}
\hline $\begin{array}{c}\text { Beam } \\
\text { Scenario }\end{array}$ & $\begin{array}{l}\text { Total CC rate } \\
/\left(10^{20} \text { pot.kT } \mathrm{T}^{F e}\right) \\
\end{array}$ & $\begin{array}{c}\text { Total CC rate } \\
/\left(\mathrm{MW} \cdot \mathrm{kT}^{F e} \cdot 10^{7} \mathrm{~s}\right) \\
\end{array}$ & $\begin{array}{c}\text { Total QE rate } \\
/\left(\mathrm{MW} \cdot \mathrm{kT}^{\mathrm{H}_{2} \mathrm{O}} \cdot 10^{7} \mathrm{~s}\right) \\
\end{array}$ \\
\hline \multicolumn{4}{|c|}{ At a distance of $735 \mathrm{~km}$} \\
\hline $\begin{array}{l}\text { NuMI LE-10 } \\
\mathrm{Z}=677, \mathrm{R}=1 \mathrm{~m}\end{array}$ & 82 & 427 & 56 \\
\hline \multicolumn{4}{|c|}{ At a distance of $1297 \mathrm{~km}$} \\
\hline $\begin{array}{l}\text { WBLE } 120 \mathrm{GeV} \\
\mathrm{Z}=380, \mathrm{R}=2 \mathrm{~m}\end{array}$ & 44 & 228 & 27.4 \\
\hline $\begin{array}{l}\text { WBLE } 60 \mathrm{GeV} \\
\mathrm{Z}=380, \mathrm{R}=2 \mathrm{~m}\end{array}$ & 16 & 164 & 25.1 \\
\hline $\begin{array}{l}\text { WBLE } 40 \mathrm{GeV} \\
\mathrm{Z}=380, \mathrm{R}=2 \mathrm{~m}\end{array}$ & 7.6 & 120 & 21.5 \\
\hline $\begin{array}{l}\text { WBLE } 28 \mathrm{GeV} \\
\mathrm{Z}=380, \mathrm{R}=2 \mathrm{~m} \\
\end{array}$ & 3.6 & 80 & 17.3 \\
\hline $\begin{array}{l}\text { WBLE } 28 \mathrm{GeV} \\
\mathrm{Z}=180, \mathrm{R}=2 \mathrm{~m}\end{array}$ & 2.6 & 58 & 13.5 \\
\hline $\begin{array}{l}\text { AGS } 28 \mathrm{GeV} \\
\mathrm{Z}=180, \mathrm{R}=2 \mathrm{~m}\end{array}$ & 3.5 & 78 & 16.2 \\
\hline
\end{tabular}

the 1MW $28 \mathrm{GeV}$ AGS beam coupled with a $500 \mathrm{kT}$ far detector using a $0.5 \mathrm{MW} 60 \mathrm{GeV}$ MI beam coupled with a $300 \mathrm{kT}$ far detector - if the neutral current backgrounds from the high energy tails can be kept under control.

The neutrino fluxes for the $28 \mathrm{GeV}$ AGS, and 40-120 GeV WBLE beams, were used as input into the GLoBES [17] software system to simulate a VLBNO experiment from FNAL to The Homestake Mine using the Water Cerenkov detector response model described in [18]. The spectrum of signal and background events for $\nu_{\mu}$ disappearance with and without, 
oscillations is shown in Figure 22. The background shown is from the process $\nu_{\mu}+N \rightarrow N^{\prime} \mu \pi$ where the pion is undetected. A sample spectrum of signal and background events in the far detector for $\nu_{\mu} \rightarrow \nu_{e}$ appearance for a value of $\sin ^{2} \theta_{13}=0.05$ is shown in Figure 23. The background for the $\nu_{e}$ appearance signal is mostly from neutral current interactions with a $\pi^{0}$ in the final state that is reconstructed as an electron, and the $\nu_{e}$ contamination from the beam. We find that the $\nu_{e}$ appearance signal is clearly visible above these backgrounds for both the $28 \mathrm{GeV}$ AGS beam and the 40,60 and $120 \mathrm{GeV}$ WBLE beams. The signal to background ratio gets progressively worse as the beam energy increases varying from 1.9:1 for the AGS $28 \mathrm{GeV}$ beam to the worst performance at $120 \mathrm{GeV}$ where its 0.8:1. A more detailed study of the VLBNO $\nu_{e}$ appearance signal and backgrounds with the AGS 28 $\mathrm{GeV}$ beam in a Water Cerenkov detector has been carried out using the SuperKamiokande detector MC as described in reference [19]. It is to be noted that we have not included the backgrounds from the processes $\nu_{\mu} \rightarrow \nu_{\tau}, \nu_{\tau}+N \rightarrow \tau+N^{\prime}+X, \tau \rightarrow e / \mu / \pi^{0}+X$ in our GLoBES analysis of the neutrino signal and background spectra observed at the far detector. The $\tau$ production threshold from $\nu_{\tau}$ is $3.5 \mathrm{GeV}$. WBLE beams produced with energies $>40$ $\mathrm{GeV}$ have significant $\nu_{\mu}$ flux contributions above the $\tau$ production threshold. Decays of $\tau$ to $e$ and hadronic modes containing $\pi^{0}$ could constitute a significant background for $\nu_{\mu} \rightarrow \nu_{e}$ appearance searches using the higher energy WBLE beams. Until a careful estimation of the $\nu_{\tau}$ background has been performed, the $\nu_{e}$ appearance and $\delta_{C P}$ sensitivity using the $120 \mathrm{GeV}$ WBLE beam remains unknown. We are currently undertaking a study of the effectiveness of adding a beam plug between the two WBLE horns in the 60 and $120 \mathrm{GeV}$ to reduce the high energy tail in the produced flux which would significantly reduce the unknown backgrounds from $\nu_{\tau} \rightarrow \tau+X$ production.

A more detailed study of the physics signals, backgrounds, and mixing parameter sensitivities of the VLBNO GLOBES simulation can be found in [18]. 

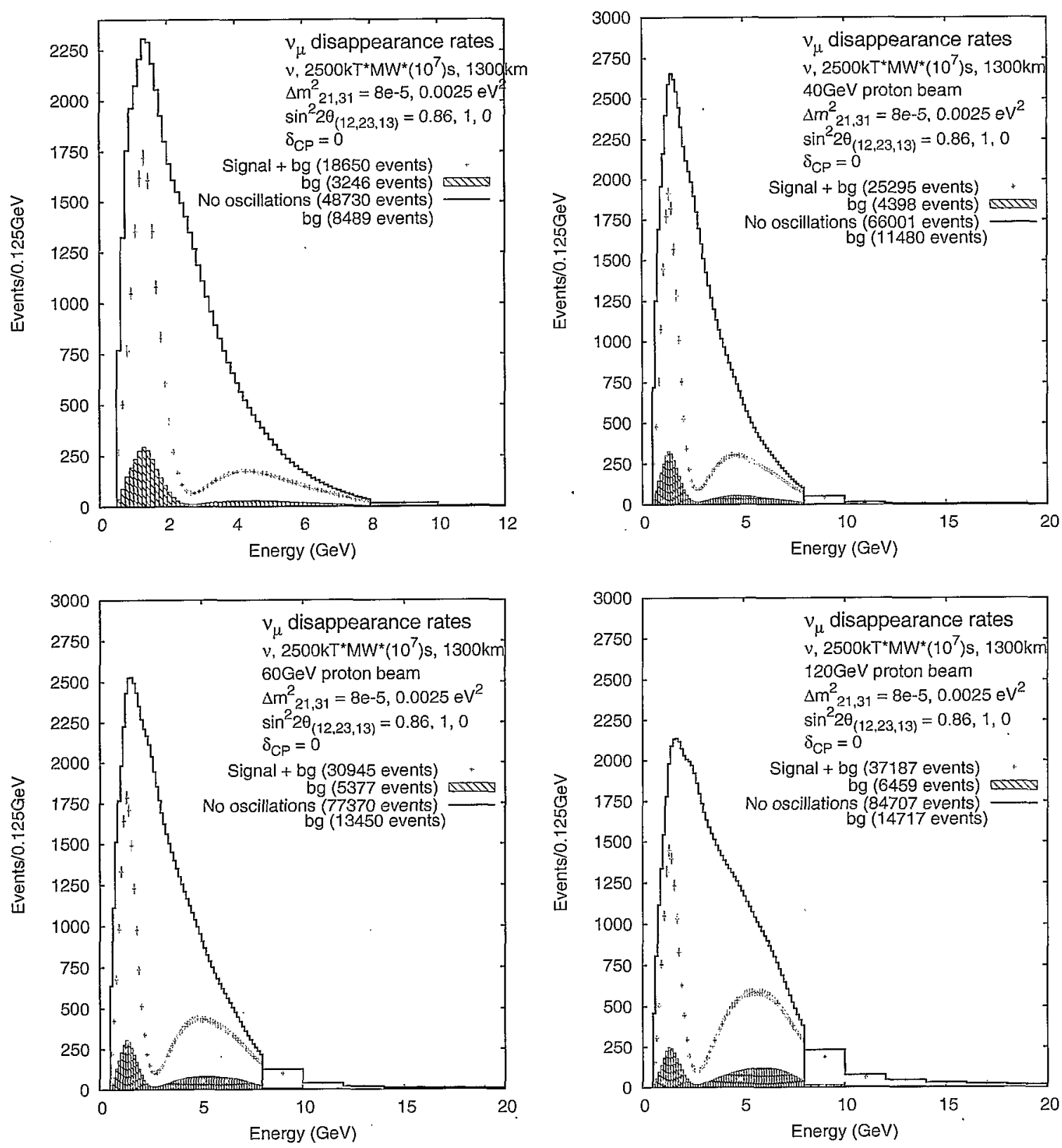

FIG. 22: The $\nu_{\mu} \rightarrow \nu_{\mu}$ survival spectrum at a Water Cerenkov detector $1297 \mathrm{~km}$ from the WBLE target for the $28 \mathrm{GeV}$ AGS beam (top left), the $40 \mathrm{GeV}$ WBLE beam (top right), the $60 \mathrm{GeV}$ WBLE beam (bottom left) and the $120 \mathrm{GeV}$ WBLE beam (bottom right). The plots are from a VLBNO experimental simulation using GLoBES. The solid histogram is the observed spectrum of events with no oscillations, the data points are the spectrum after oscillations. The hatched histogram is the background to the oscillated signal from charged-current single pion production where the pion is undetected. The assumed exposure is 2500 kT.MW. $10^{7}$ seconds. 

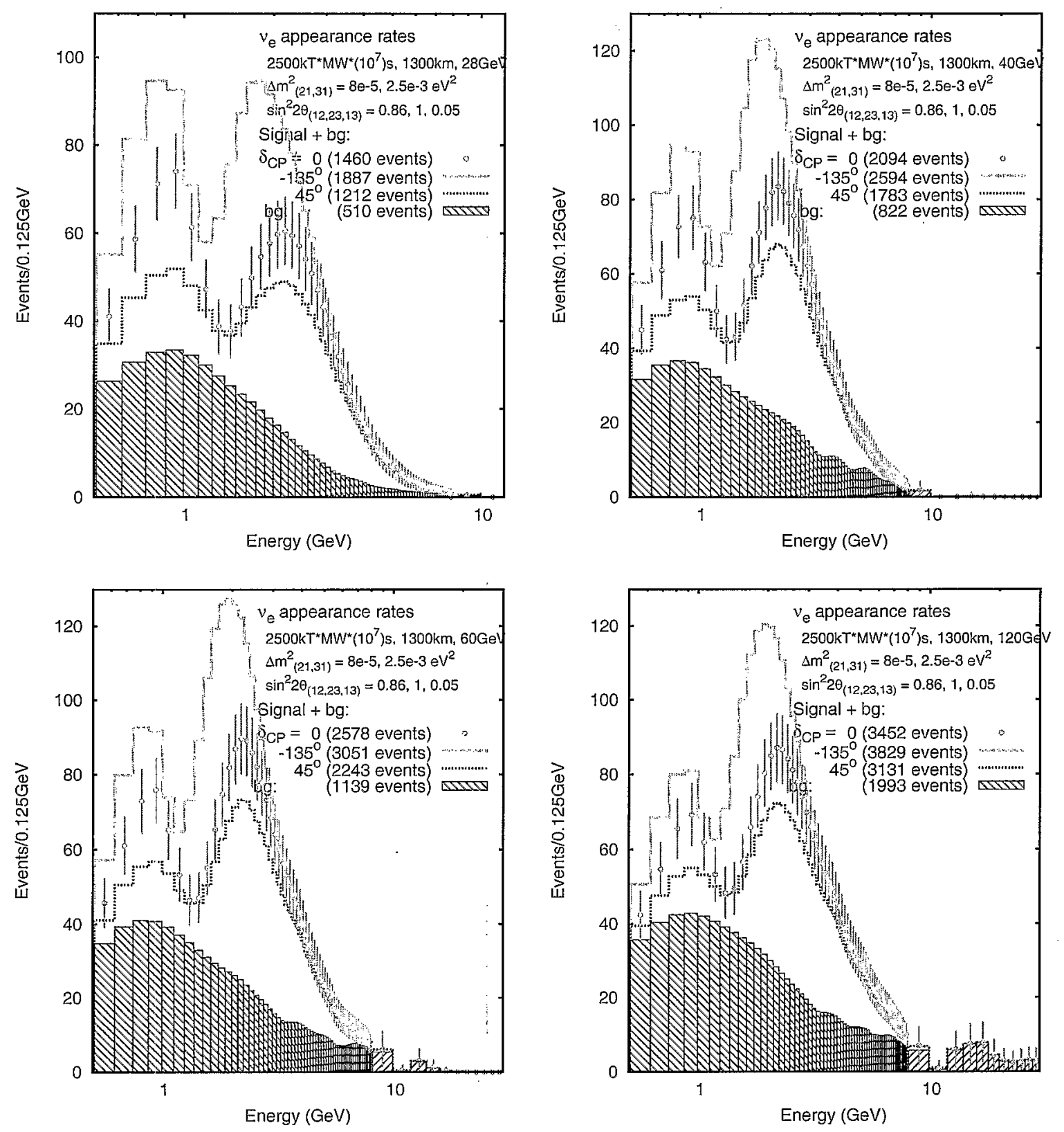

FIG. 23: The $\nu_{\mu} \rightarrow \nu_{e}$ appearance spectrum at a Water Cerenkov detector $1297 \mathrm{~km}$ for the $28 \mathrm{GeV}$ AGS beam (top left), the $40 \mathrm{GeV}$ WBLE beam (top right), the $60 \mathrm{GeV}$ WBLE beam (bottom left) and the $120 \mathrm{GeV}$ WBLE beam (bottom right). The plots are from a VLBNO experimental simulation unsing GLoBES with $\sin ^{2} 2 \theta_{13}=0.05$. The hatched histogram is the spectrum of the background from neutral current events and beam $\nu_{\epsilon}$. The background from $\nu_{\mu} \rightarrow \nu_{\tau}$ oscillated events IS NOT INCLUDED. The data points are the spectrum of observed $\nu_{e}$ events for $\delta_{C P}=0$, and the two dotted histograms are the observed spectra for $\delta_{C P}=-135^{\circ}, 45^{\circ}$. The assumed exposure is 2500 kT.MW. $10^{7}$ seconds. 


\section{SUMMARY}

We have implemented a simulation of a Wide-Band Low-Energy (WBLE) neutrino beam using a new beam-line sited at Fermi National Lab and directed towards a possible DUSEL site. We use the FLUKA '05 particle transport code to model the hadron production spectra obtained from a proton beam on a graphite target. We have used the NuMI GEANT (v3.21) based simulation framework to simulate the hadron transport from the FLUKA '05 target simulation through the BNL AGS horn geometries and decay pipe. We have a preliminary determination of the possible decay volume geometries based on the conceptual layout of a Fermilab to DUSEL neutrino beam-line utilizing the NuMI extraction region. We have studied the neutrino flux at $1 \mathrm{~km}$ and $\mathrm{CC}$ event rates at the far detector for different MI beam energies, horn currents and decay volume geometries. The simulation has been validated by comparing to the NuMI beam-line results and to the previous AGS design. The real-life performance of the NuMI beam-line simulation and flux prediction is now well understood from the analysis of 1 year of data collected by the MINOS experiment's near detector. We have produced a preliminary estimate of the event rates observable at a DUSEL detector. The signal and background spectra observed at DUSEL using the 40-60 GeV WBLE beam for some sample oscillation parameters have been calculated using the GLoBES software system.

We find that it may be possible to reach the design sensitivities of a VLBNO experiment using a 40-60 GeV WBLE beam from Fermilab to DUSEL with lower power ( $<=1 \mathrm{MW}$ ) and smaller far detector than previously thought, provided the $\mathrm{NC}$ and $\tau$ backgrounds from the larger high energy neutrino tails are kept under control. The advantage of being able to run with only $500 \mathrm{KW}$ beam power at $40-60 \mathrm{GeV}$ is that such power levels can be achieved with incremental upgrades to the current Fermilab accelerator complex and without the need for an expensive Proton Driver.

The estimation of the physics sensitivities using the Fermilab WBLE on-axis neutrino spectra produced from our preliminary beam-line simulation is discussed in [18]. 


\section{Acknowledgments}

We thank the Fermilab staff and the technical staffs of the participating institutions for their vital contributions. We especially thank the Fermilab NuMI staff for their valuable feedback, and the MINOS collaboration for use of their GEANT based neutrino beamline simulation. This study would not have been possible without the remarkable efforts of the BNL AGS staff and BNL physicists which resulted in the highly effective designs of the neutrino beamline components used in this study. Special thanks to Milind Diwan for his encouragement and guidance. This work was supported by the U.S. Department of Energy and National Science Foundation. 


\section{APPENDIX A: HORN CURRENT OPTIMIZATION STUDIES}

We have studied the effect of running the WBLE horns at $200 \mathrm{kA}$ instead of the $250 \mathrm{kA}$ current. This should increase the lifetime of the horns, and matches the experience gained from the NuMI horns which have been shown .to run reliably at $200 \mathrm{kA}$. The results are shown in Figure 24. We find a decrease of $11 \%$ and $8 \%$ in the total neutrino flux and the integrated flux $<5 \mathrm{GeV}$ respectively for both 40 and $60 \mathrm{GeV}$ beams. We find a decrease of $15 \%$ in the total $\nu$ CC rates in the far detector when going to the lower horn current of 200 $\mathrm{kA}$ for both beam energies of 40 and $60 \mathrm{GeV}$.

\section{WBLE, horn current variation}

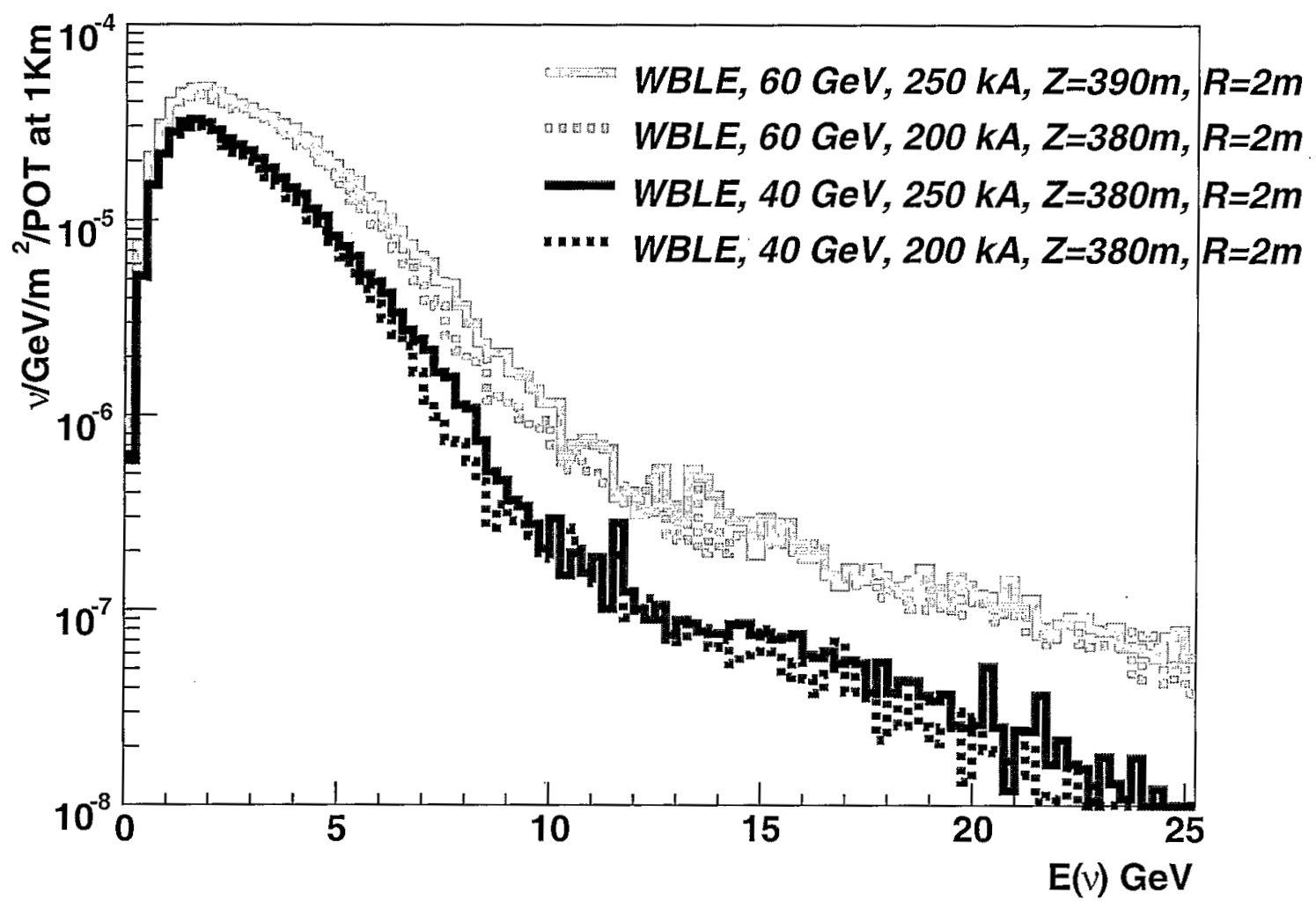

FIG. 24: WBLE 40 and $60 \mathrm{GeV}$ beam neutrino fluxes using different horn currents. 
[1] M. Diwan et. al. Phys. Rev. D. 68, 012002 (2003)

[2] M. Diwan, "The Case for a Super Neutrino Beam". Conference Proceedings of Heavy Quarks and Leptons, 2004. San Juan, Puerto Rico, June 1-5 (2004). hep-ex/0407047

[3] J. Hylen et. al.." Conceptual design for the technical components of the neutrino beam for the main injector (NuMI).", FERMILAB-TM-2018 Sep (1997).

The NuMI Technical Design Handbook is available at http://www-numi.fnal.gov/numwork/tdh/tdh_index.html

[4] D. G. Michael et. al. "Observation of muon neutrino disappearance with the MINOS detectors in the NuMI neutrino beam". hep-ex 0607088, FERMILAB-PUB-06-243, BNL-76806-2006-J A.

[5] R. Brun et al., CERN-DD-78-2-REV

[6] J.A.lessi et. al. "The AGS Super Neutrino Beam Facility Conceptual Design Report". BNL73210-2004-IR, October 8, (2004)

[7] Deep Underground Science and Engineering Laboratory, NSF http://www.dusel.org/ .

[8] A. Marchionni, "Main Injector and NuMI upgrades and beyond". Presentation at the Workshop on Long Baseline Neutrino experiments, Fermilab, Batavia, IL, March 6-7 (2006).

[9] G. Bock, "Fermilab Proton Beams: Program Perspectives". Presentation at the Science and Engineering Henderson-DUSEL Capstone Workshop, Stony Brook, NY May 5 (2006).

[10] Private communication with Dixon Bogert and Wes Smart, Fermilab Accelerator Division, 2006.

[11] R.D. Kephart et. al. Nucl. Phys. Proc. Suppl. 147:41-44 (2005)

G. Apollinari et. al. Nucl. Phys. Proc. Supll. 155:61-66 (2006)

[12] R. Zwaska, "Fermilab Proton Projections for Long Baseline Neutrino Beam". Report prepared for the Joint FNAL-BNL Very Long Baseline Neutrino Program Study, 2006.

[13] A. Fasso', A. Ferrari, J. Ranft, and P.R. Sala, "FLUKA: a multi-particle transport code", CERN Yellow Report (2005), INFN/TC_05/11, in press.

A. Fasso', A. Ferrari, S. Roesler, P.R. Sala, G. Battistoni, F. Cerutti, E. Gadioli, M.V. Garzelli, F. Ballarini, A. Ottolenghi, A. Empl and J. Ranft, "The physics models of FLUKA: status 
and recent developments", Computing in High Energy and Nuclear Physics 2003 Conference (CHEP2003), La Jolla, CA, USA, March 24-28, 2003, (paper MOMT005) eConf C0303241 (2003), arXiv:hep-ph/0306267

[14] D. Jaffe et. al. "Comparison of inclusive particle production in $14.6 \mathrm{GeV} / \mathrm{c}$ proton-nucleus collisions with simulation", Nuclear Inst. and Methods in Physics Research, B, 28 Feb (2006). BNL-75079-2005-JA. hep-ex/0511012. http://dx.doi.org/10.1016/j.nimb.2006.01.014

[15] N. Simos et. al. "Target System for a Long Baseline Neutrino Beam". Report prepared for the Joint FNAL-BNL Very Long Baseline Neutrino Program Study, 2006. http://nwg.phy.bnl.gov/ diwan/nwg/fnal-bnl/

[16] L. A. Ahrens et. al. Phys. Rev. D 34, 75-84 (1986).

[17] P. Huber, M. Lindner, and W. Winter. "Simulation of long-baseline neutrino oscillation experiments with GLoBES". hep-ph/0407333 (2004).

[18] V. Barger et. al. "Precision Physics with a Wide Band Super Neutrino Beam", hepph/0607177. BNL-76797-2006-JA.

[19] C. Yanagisawa et. al. "A Study of Background for Detecting Appearance Signal of Neutrino Oscillation $\nu_{\mu} \rightarrow \nu_{e}$ Using a Very Long Baseline Wideband Neutrino Beam and a Large Underground Water Cerenkov Detector". Report prepared for the Joint FNAL-BNL Very Long Baseline Neutrino Program Study, 2006. http://nwg.phy.bnl.gov/ diwan/nwg/fnal-bnl/

[20] The NuMI proton pulse is divided into 6 batches, each batch corresponds to a single extraction from the Fermilab booster to the Main Injector

[21] We used a horn current of $200 \mathrm{kA}$ in this study. 